\title{
FAM96A and FAM96B Suppress Breast Cancer Proliferation and Migration via the Wnt/ $\beta$-Catenin Signaling Pathway
}

\section{Di-Di Zhang}

Beijing University of Technology

Xiao-Lin Sun

Beijing University of Technology

Zhao-Yuan Liang

Beijing University of Technology

Li-Na Zhang ( $\nabla$ Inzhang@bjut.edu.cn )

Beijing University of Technology https://orcid.org/0000-0003-0232-8766

\section{Research}

Keywords: FAM96A, FAM96B, Wnt/ $\beta$-catenin, tumor suppressor, breast cancer

Posted Date: August 31st, 2021

DOI: https://doi.org/10.21203/rs.3.rs-823596/v1

License: (a) (i) This work is licensed under a Creative Commons Attribution 4.0 International License. Read Full License 


\section{Abstract}

Background: Family with sequence similarity 96 member A and B (FAM96A and FAM96B) are two highly conserved homologous proteins belonging to MIP18 family. Many studies have shown that FAM96A and FAM96B play many different functions mainly through interacting with other different proteins. Recently, several studies show that FAM96A and FAM96B are significantly down-regulated compared in human gastrointestinal stromal tumors, colon cancer, liver cancer and gastric cancer with corresponding normal tissues. However, the molecular regulatory mechanisms of FAM96A and FAM96B in breast cancer development and metastasis are still unclear. In this work, we aimed to explore the molecular mechanisms of FAM96A and FAM96B in breast cancer progression.

Methods: We used specific siRNAs to down-regulate FAM96A and FAM96B expression, and used recombinant plasmids to up-regulate FAM96A and FAM96B expression in breast cancer cells. Cell proliferation was measured using MTT and colony formation assays. Cell cycle and apoptosis were detected by flow cytometry analysis. Wound healing and transwell assays were used to examine cell migration and invasion abilities. The relationships among FAM96A/B, EMT and Wnt/ $\beta$-catenin signaling pathway were determined by analyzing the expression changes of classical markers and biological functional changes after XAV-939 inhibitor treatment.

Results: We found that FAM96A and FAM96B expression in breast cancer was down-regulated. FAM96A/B overexpression suppressed breast cancer cell proliferation, invasion and migration, induced cell apoptosis and led to cell cycle arrested in G0/G1 phase. Conversely, FAM96A/B knockdown exhibited the opposite effects on breast cancer cells. Moreover, our data demonstrated that FAM96A/B overexpression suppressed EMT and Wnt/ $\beta$-catenin signaling pathway, while FAM96A/B knockdown showed the promoting effects on EMT and Wnt/ $\beta$-catenin signaling pathway in breast cancer cells. Furthermore, a Wnt pathway inhibitor, XAV-939 treatment reversed the promoting effects of FAM96A and FAM96B knockdown on breast cancer cell proliferation, invasion and migration.

Conclusions: Our findings revealed that FAM96A and FAM96B may act as tumor suppressor genes and inhibit breast cancer progression via modulating the Wnt/ $\beta$-catenin pathway, which can provide the potential markers for the diagnosis and treatment of breast cancer.

\section{Background}

Breast cancer is the most frequent and malignant cancer in women worldwide and very easy to metastasize and relapse, which is also the main cause of death in breast cancer patients. In recent years, with the rapid development of society and the change of people's life style, the incidence rate of breast cancer worldwide has significantly increased [1]. However, the underlying molecular mechanisms of breast cancer occurrence and metastasis are still not fully elucidated. Currently, the molecular targeted therapy has been considered an effective approach for breast cancer treatment. Therefore, it is very urgently needed to discover the novel diagnostic markers or therapeutic targets for breast cancer therapy. 
Family with sequence similarity 96 member $A$ (FAM96A) is also called cytosolic iron-sulfur assembly component 2A (CIAO2A), and family with sequence similarity 96 member $B$ (FAM96B) is also called cytosolic iron-sulfur assembly component 2B (CIAO2B) or MIP18. FAM96A and FAM96B are ubiquitously expressed and evolutionarily highly conserved proteins that both of them contain a common domain of unknown function 59 (DUF59). In terms of their primary structure, FAM96A contains 160 amino acids and FAM96B contains 163 amino acids, and the molecular weight of these two proteins is about $18 \mathrm{kDa}$. Sequence alignment results show that FAM96A and FAM96B share $50 \%$ sequence identity, but the sequence variation is that FAM96A protein has a predicted signal sequence at the $\mathrm{N}$-terminal whereas FAM96B does not. Results of yeast two-hybrid and immunoprecipitation experiments demonstrate that both FAM96A and FAM96B interact directly with cytosolic iron-sulfur assembly component 1 (CIAO1), and they are the components of the cytosolic iron-sulfur protein assembly (CIA) complex and involved in the cytosolic iron-sulfur protein assembly [2, 3]. FAM96A or FAM96B interacts with CIAO1 and the CIAtargeting factor MMS19 to form two large different protein complexes, respectively. FAM96A-CIA01MMS19 complex regulates the cellular iron homeostasis by promoting the maturation of iron regulatory protein 1 (IRP1) and the stabilization of iron regulatory protein 2 (IRP2), while FAM96B-CIA01-MMS19 complex facilitates the assembly of most cytosolic-nuclear iron-sulfur proteins [4]. Even more interesting is that the FAM96A-CIA01 complex play a functional role in the maturation of aconitase [5]. FAM96B not only interacts with CIAO1 and MMS19, but also further interacts with ADP/ATP transposase 2 (ANT2) to form a large complex to participate in the cytosolic iron-sulfur cluster proteins assembly [6]. In addition, FAM96B and CIA01 are also shown to be the components of the MMXD complex (MIP18-MMS19-XPD), which is responsible for DNA repair and correct chromosome segregation. Silencing the expression of FAM96B in the human colon cancer HCT116 cells results in the wrong localization of mitotic spindle and the accumulation of heterotypic nuclei, which indicates that FAM96B is distributed in the mitotic spindle and plays a key role in the normal separation of mitotic chromosomes [7]. Except for these important functions of FAM96A, another study has shown that FAM96A mRNA is highly enriched in human and mouse macrophages, and its expression level is significantly higher than that of $B$ and $T$ lymphocytes [2]. A recent study in the mouse colitis model has discovered that FAM $96 \mathrm{~A}^{-/-}$knockout mice are significantly more susceptible to dextran sulfate sodium (DSS)-induced colitis, and FAM96A can maintain the homeostasis of colonic environment by preventing intestinal microbial imbalance [8]. The latest research demonstrates that FAM96A knockout can promote the polarization of macrophages through reducing the secretion of pro-inflammatory cytokines, and thus to protect mice from sepsis death [9]. These findings provide new evidence for that FAM96A may be an important inflammatory regulatory factor and antiinflammatory drug design target.

Interestingly, many studies have proved that FAM96B plays diverse functions in vivo, which are mainly related to its interacting proteins. For example, FAM96B can down-regulate the expression of the basic helix-loop-helix protein E2-2 in endothelial cells through interacting with E2-2, and participate in the endothelial cell migration, proliferation and angiogenesis [10]. FAM96B is also proved to be a novel binding partner of selenoprotein W (SelW) in the brain, which helps to provides new evidence for clarifying the role of SelW in the brain development [11]. In addition, FAM96B is also identified to be a 
novel binding partner of prelamin A, which suggesting that the direct interaction between prelamin $A$ and FAM96B is more likely to provide some evidence for verifying the mechanism of prelamin $A$ in the premature aging [12]. Recent research reports that FAM96B plays a crucial role in suppressing the senescence of dental pulp stem cells, promoting osteogenic differentiation and proliferation. Silencing FAM96B results in a decrease in human telomerase reverse transcriptase (hTERT) activity and a significant increase in the number of senescence-related SA- $\beta$-gal positive cells, as well as the expression levels of p16 and p53 proteins [13].

Most importantly, FAM96A and FAM96B are shown to participate in the regulation of tumor cell apoptosis by interacting with different proteins. Specifically speaking, FAM96A interacts with apoptotic protease activating factor-1 (Apaf-1) and plays a key role in promoting apoptosis in human gastrointestinal stromal tumor cells through mitochondrial apoptosis pathway [14]. However, FAM96B interacts with Apoptin and acts a certain role in Apoptin-induced apoptosis of tumor cells. Overexpression of FAM96B can significantly reduce the apoptotic rate of Apoptin-induced tumor cells, which may be related to the change of nuclear localization of Apoptin induced by FAM96B [15].

In recent years, some studies have already shown that the expression levels of FAM96A and FAM96B in human gastrointestinal stromal tumors, colon cancer, liver cancer and gastric cancer are significantly down-regulated. For instance, FAM96A can possess apoptosome-activating potential through interacting with Apaf-1 and suppress the tumorigenicity of human gastrointestinal stromal tumor cells, which suggesting that FAM96A is considered to be a novel pro-apoptotic tumor suppressor in human gastrointestinal stromal tumors [14]. Subsequent studies have found that the fusion protein of human telomerase reverse transcriptase receptor (hTERTR) and FAM96A can specifically bind to hTERT and Apaf-1, respectively, induce apoptosis and further improve the apoptosis sensitivity of liver cancer cells, and thus to inhibit the liver cancer cell proliferation, migration and invasion [16]. Moreover, hTERTFAM96A combination can also promote cytotoxic $T$ lymphocyte reaction, release of interferon- $\gamma$ and inflammatory infiltration of T lymphocytes, thus enhancing the toxic effect on liver cancer cells [17]. Above all, hTERT-FAM96A fusion protein may be an effective anti-tumor inhibitor. These research work further suggest that FAM96A may be a potential tumor suppressor gene that involved in the tumor occurrence and development.

However, there is no detailed research report on the functional role of FAM96B in the tumor progression at present. It is only known that FAM96B expression is down-regulated in several types of tumor tissues according to some domestic research reports. Overexpression of FAM96B in different cancer cell lines results in the growth inhibition and induces tumor cell apoptosis, but its specific molecular mechanism in tumor progression is still unknown. Therefore, although FAM96A and FAM96B have been proved to exert diverse functions in vitro and in vivo, their functional roles and the molecular regulatory mechanisms in tumor occurrence and development are still remain unclear as so far.

In this research, we mainly investigated the functional role of FAM96A and FAM96B in cell proliferation, cell apoptosis, cell cycle, cell migration and invasion of breast cancer cells. Mechanistically, we found 
that FAM96A and FAM96B may inhibit the proliferation, invasion and migration of breast cancer cells via the suppression of EMT and Wnt/ $\beta$-catenin signaling pathway. These all findings provide new evidence that FAM96A and FAM96B act as tumor suppressor genes to suppress the breast cancer progression. It is also suggested that FAM96A and FAM96B may serve as the novel therapeutic targets and diagnostic markers for breast cancer.

\section{Materials And Methods}

\section{Antibodies}

Rabbit antibody against FAM96A was purchased from EpiGentek (Farmingdale, NY, USA). Rabbit antibody against FAM96B was obtained from Abcam (Cambridge, UK). Rabbit antibodies against p21,

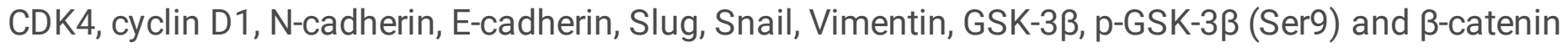
were purchased from Cell Signaling Technology (CST) (Danvers, MA, USA). Rabbit antibodies against Bcl2, Bax, Caspase 3, cyclin E, CDK2 and c-Myc were from Wanleibio (Shenyang, China). The anti- $\beta$-actin mouse antibody was from ZSGB-bio (Beijing, China). Goat anti-rabbit and goat anti-mouse HRPconjugated secondary antibodies were from ABGENT (San Diego, CA, USA).

\section{Cell lines and culture}

Human breast cancer cell lines including MCF-7 and MDA-MB-231, and the normal breast epithelial cell line MCF-10A were obtained from Chinese Academy of Sciences. These cell lines were maintained in the DMEM medium (Hyclone, Logan, UT, USA) containing 10\% FBS (Gibco, Grand Island, NY, USA) and 1\% penicillin-streptomycin (HyClone) at $37^{\circ} \mathrm{C}$ in a humidified cell culture incubator with $5 \% \mathrm{CO}_{2}$.

\section{Cell transfection}

The specific siRNAs against FAM96A (siFAM96A), FAM96B (siFAM96B) and non-specific control siRNA (siNC) were designed and synthesized to down-regulate FAM96A and FAM96B expression by Genepharm Co., Ltd (Shanghai, China). The specific siRNA sequences targeting FAM96A and FAM96B were shown in the Supplementary Table S1. The CDNA sequences of FAM96A and FAM96B were subcloned into the pEGFP-C3 vector to up-regulate FAM96A and FAM96B expression by PCR analysis. The transfection reagent lipofectamine ${ }^{\circledR} 3000$ (Invitrogen, Waltham, MA, USA) was used to transfect with siRNAs or recombinant plasmids into MCF-7 and MDA-MB-231 cells according to the manufacturer's instructions. The overexpression and knockdown efficiencies of FAM96A and FAM96B in the mRNA and protein levels were detected using RT-qPCR and Western blot assays, respectively.

For specific inhibition of the Wnt/ $\beta$-catenin signaling pathway, XAV-939, a potent tankyrase inhibitor that targets Wnt/ $\beta$-catenin signaling, was added into the DMEM medium of the cultured breast cancer cells with a final concentration of $30 \mathrm{nM}$ for $24 \mathrm{~h}$ (Selleck Chemicals, Houston, TX, USA).

\section{RT-qPCR}


Total RNA was extracted from the cultured cells using a RNAprep Pure Cell Kit (TIANGEN, Beijing, China), and $1 \mu \mathrm{g}$ total RNA was reversed transcribed into cDNA with PrimeScript ${ }^{\mathrm{TM}}{ }_{\mathrm{RT}}$ reagent $K$ it with gDNA Eraser (Takara, Dalian, China). RT-qPCR was used to detect the gene expression by SYBR ${ }^{\circledR}$ Premix DimerEraser Kit (Takara) according to the manufacturer's protocols. The relative mRNA level of each gene was calculated using $2^{-\triangle \triangle \mathrm{Ct}}$ method and normalized by $G A P D H$ mRNA. The specific primer sequence of each gene used in this experiment are shown in the Supplementary Table S2.

\section{Western blot}

Total proteins were extracted from the cultured cells using RIPA buffer supplemented with PMSF and cocktail protease inhibitor (Genview, Beijing, China). The concentration of each total protein sample was measured by a BCA Protein Assay Kit (Genview). Each cell sample containing $40 \mu \mathrm{g}$ protein were subjected to separate by $10 \%$ SDS-polyacrylamide gel, and then the proteins were transferred onto PVDF membranes (Millipore, Billerica, MA, USA). After being blocked in 5\% non-fat skim milk for $2 \mathrm{~h}$ at room temperature, the PVDF membranes were incubated with corresponding primary antibodies at $4{ }^{\circ} \mathrm{C}$ overnight. The primary antibodies used in this experiment included anti-FAM96A, anti-FAM96B, anti-p21, anti-cyclin D1, anti-CDK4, anti-cyclin E, anti-CDK2, anti-Bax, anti-Bcl-2, anti-Caspase-3, anti-N-cadherin, anti-Vimentin, anti-E-cadherin, anti-Snail, anti-Slug, anti- $\beta$-catenin, anti-GSK-3 $\beta$, anti-p-GSK-3 $\beta$, and anti-CMyc (1:500 1:1000 dilution). $\beta$-actin was served as the protein loading control (1:5000 dilution). Subsequently, the PVDF membranes were washed three times for $10 \mathrm{~min}$, and then incubated with the appropriate HRP-conjugated secondary antibodies for $1 \mathrm{~h}$ at room temperature. Finally, the protein bands were detected by ECL chemiluminescent HRP substrate (Millipore) and visualized with a Tanon 4200 chemiluminescence imaging system (Tanon, Shanghai, China).

\section{Cell proliferation assay}

Cells were seeded into a 96-well plate with $1 \times 10^{3}$ cells per well. Before daily measurement, $10 \mu$ MTT solution (Solarbio, Beijing, China) was added into the each well of the 96-well plate at the different time points ( $24 \mathrm{~h}, 48 \mathrm{~h}, 72 \mathrm{~h}, 96 \mathrm{~h}$ ). After incubation for another $4 \mathrm{~h}$ at $37^{\circ} \mathrm{C}$, the absorbance was measured at $490 \mathrm{~nm}$ for the MTT solution using a microplate reader (BioTek, Winooski, VT, USA). All experiments were performed in triplicate.

\section{Colony formation assay}

Cells were seeded into a 6-well plate with $1 \times 10^{3}$ cells per well and cultured for about 14 days. After obvious colonies were formed, the culture medium was removed and the colonies were fixed with $4 \%$ paraformaldehyde (Genview) for $30 \mathrm{~min}$. Subsequently, the colonies were stained with $0.1 \%$ crystal violet (Amresco, Solon, $\mathrm{OH}, \mathrm{USA}$ ) for $30 \mathrm{~min}$. After washing and drying, the stained colonies were photographed and counted manually. The statistical results are the mean values of three independent experiments.

\section{Cell cycle assay}


Cells were harvested by trypsin digestion, washed with PBS and fixed with $70 \%$ ethanol in PBS at $4{ }^{\circ} \mathrm{C}$ overnight. Next, the fixed cells were treated with $100 \mu \mathrm{g} / \mathrm{ml}$ RNase A (TIANGEN) in PBS for 30 min at 37 ${ }^{\circ} \mathrm{C}$, and then stained with $100 \mu \mathrm{g} / \mathrm{ml}$ propidium iodide (PI) solution (Solarbio) for $30 \mathrm{~min}$ at room temperature. Cell cycle progression was detected by FACSCalibur flow cytometer (BD Biosciences, San Jose, CA, USA), and the number of cells in G0/G1, S and G2/M phase were assayed with ModFit LT 3.0 software.

\section{Cell apoptosis assay}

Annexin V-FITC/PI or Annexin V-FITC/7-AAD Apoptosis Detection Kit (Solarbio, Beijing, China) was used to evaluate cell apoptosis. In brief, the harvested cells were incubated with $5 \mu$ I Annexin V-FITC and $5 \mu$ I PI or 7-AAD in $100 \mu$ l binding buffer for 10 min according to the manufacturer's protocols in the darkness. Then, cell apoptosis was analyzed by FACSCalibur flow cytometer (BD Biosciences). The results of cell apoptosis were analyzed with FlowJo v10 software. The statistical data displayed mainly included the proportions of apoptotic cells and survival cells.

\section{Wound healing assay}

The wound was scratched with a $200 \mu$ sterile pipette tip when cells were grown to $70 \sim 80 \%$ confluence in 12-well plates. After washing with PBS, the cells were cultivated for $24 \mathrm{~h}$ and $48 \mathrm{~h}$ in the DMEM medium containing 5\% FBS, respectively. The images were obtained using an inverted optical microscope (Olympus IX71, Tokyo, Japan), and the relative migration rate of cells assessed by calculating the changes of scratch width using Image-Pro Plus 6.0 software. The experiments were performed in triplicate.

\section{Transwell assay}

Transwell migration and invasion assays were assessed using 24-well transwell chambers (Millipore, Billerica, MA, USA). For transwell cell migration assay, $5 \times 10^{4}$ cells were resuspended with $200 \mu$ l serumfree DMEM medium and seeded into the upper 24-well transwell chamber. For transwell cell invasion assay, $8 \times 10^{4}$ cells were resuspended with $200 \mu$ l serum-free DMEM medium and plated on the matrigel (BD Biosciences) pre-coated 24-well transwell chambers. The lower tranwell chamber was filled with $500 \mathrm{ml}$ DMEM medium containing 20\% FBS. After being cultured for $24 \mathrm{~h}$ at $37^{\circ} \mathrm{C}$, non-migrated cells and non-invaded cells in the upper surface of the transwell chamber were slightly wiped with a cotton tip, while the migrated cells and invaded cells on the lower surface of the 24-well transwell chamber were fixed using $4 \%$ paraformaldehyde and stained with $0.5 \%$ crystal violet for $30 \mathrm{~min}$. The number of migrated cells and invaded cells was photographed and counted under an inverted microscope (Olympus IX71). These experiments were performed in triplicate.

\section{Bioinformatics analysis}


The expression changes of $F A M 96 A$ and $F A M 96 B$ mRNA levels in various type of cancers including breast cancer was analyzed using Oncomine online database (https://www.oncomine.org/resource/login.html), which is a cancer microarray database and online datemining platform [18]. The specific mRNA expression level of FAM96A and FAM96B between invasive breast carcinoma and normal breast tissue was analyzed in a specific dataset of breast cancer, respectively.

\section{Statistical analysis}

All the data analysis was performed using GraphPad Prism 6.0 (GraphPad Software Inc., La Jolla, CA, USA). All the experiments were performed in triplicate. All the measured data were expressed as mean \pm SEM. The statistically significance of the data between two independent groups was calculated using unpaired two-tailed Student's $t$ test. Difference with ${ }^{*} p<0.05$ was considered to be statistically significant.

\section{Results}

\section{FAM96A and FAM96B expression is down-regulated in breast cancer}

Previous study has reported that FAM96A was down-regulated in human gastrointestinal stromal tumors and was a novel pro-apoptotic tumor suppressor [14]. We first analyzed the differential expression levels of FAM96A and FAM96B in breast cancer using Oncomine online database (https://www.oncomine.org/resource/login.html). The data of the online cancer tissue microarray analysis showed that the mRNA levels of FAM96A and FAM96B in breast cancer tissues were significantly lower than that in normal breast tissues (Fig. 1A). Moreover, we analyzed the mRNA expression of $F A M 96 A$ and $F A M 96 B$ between invasive breast carcinoma and normal breast tissue according to the specific breast cancer datasets from Oncomine database. The results showed that FAM96A expression in invasive breast carcinoma was 7 folds lower than that in normal breast tissue in Finak Breast dataset, while FAM96B expression in invasive breast carcinoma was about 1 fold lower than that in normal breast tissue in Gluck Breast dataset (Fig. 1B). To further confirm the low expression of FAM96A and FAM96B in breast cancer, we also investigated the mRNA and protein expression levels of FAM96A and FAM96B in the two breast cancer cell lines including MCF-7 and MDA-MB-231 and normal breast epithelial cell line MCF-10A using RT-qPCR and Western blot assays. Our experimental results showed that both the mRNA and protein expression levels of FAM96A and FAM96B in MCF-10A cells were obviously higher than that in MCF-7 and MDA-MB-231 breast cancer cells (Fig. 1C, D). In general, these results confirm that the expression of FAM96A and FAM96B is indeed down-regulated in breast cancer.

\section{FAM96A and FAM96B inhibit breast cancer cell proliferation}

In order to investigate the specific biological functions of FAM96A and FAM96B in breast cancer progression, gain-of-function and loss-of-function experiments were simultaneously performed in MCF-7 and MDA-MB-231 breast cancer cell lines. FAM96A and FAM96B specific cDNA recombinant plasmids 
were used to up-regulate the expression of FAM96A and FAM96B in MCF-7 and MDA-MB-231 cells (Fig. 2A, B). Specific siRNAs were used to down-regulate the expression of FAM96A and FAM96B in MCF-7 and MDA-MB-231 cells (Fig. 2C, D). According to the knockdown efficiencies of the two siRNAs targeting FAM96A and FAM96B, we next selected siRNA-2 targeting FAM96A and siRNA-1 targeting FAM96B for the subsequent functional experiments, respectively. We first analyzed the effects of FAM96A and FAM96B on the proliferation abilities of MCF-7 and MDA-MB-231 cells though MTT and colony formation assays. Our results of MTT assay suggested that overexpression of FAM96A and FAM96B remarkably led to the inhibitory proliferation abilities of MCF-7 and MDA-MB-231 cells, while knockdown of FAM96A and FAM96B promoted the proliferation abilities of MCF-7 and MDA-MB-231 cells (Fig. 2E). Similarly, results of colony formation assay indicated that the number of colonies was significantly decreased when FAM96A and FAM96B expression was up-regulated, while the number of colonies increased when FAM96A and FAM96B expression was down-regulated (Fig. 2F). Taken together, these findings suggest that FAM96A and FAM96B play an inhibitory effect on the proliferation abilities of breast cancer cells.

\section{FAM96A and FAM96B retard breast cancer cell cycle}

To explore the underlying mechanisms of the inhibitory effect of FAM96A and FAM96B on breast cancer proliferation, we measured the cell cycle using propidium iodide (PI) staining. Flow cytometry was used to analyze the proportions of cells at different cell cycle phases. As shown in Fig. 3A and B, overexpression of FAM96A and FAM96B resulted in a significant elevation in the proportion of G0/G1 phase cells accompanied with an obvious reduction in the proportion of $S$ phase cells compared with that of control cells, while knockdown of FAM96A and FAM96B exhibited a reversed effect on the proportion of G0/G1 and $\mathrm{S}$ phases cells. These results indicate that FAM96A and FAM96B overexpression block breast cancer cell cycle progression at G0/G1 phase.

It is generally accepted that p21 is a major negative regulator of G1-S phase transition and a potent inhibitor of cyclin-dependent kinases (CDKs) [19]. So, we then examined the protein expression changes of $\mathrm{G} 1$ phase-related proteins including p21, CDK4, cyclin D1, CDK2 and cyclin E through western blotting. Consistently, our results showed that overexpression of FAM96A and FAM96B led to a significant increase of p21 and an obvious decrease of cyclin D1/CDK4 and cyclin E/CDK2 complexes. In contrast, knockdown of FAM96A and FAM96B dramatically decreased the expression of p21 and increased the expression of cyclin D1/ CDK4 and cyclin E/CDK2 in both MCF-7 and MDA-MB-231 breast cancer cells (Fig. 3C). Collectively, these data conclude that the inhibitory growth effects and the cell cycle arrest at G0/G1 phase of FAM96A and FAM96B overexpression may be caused by the expression changes of G1 phase-related proteins.

\section{FAM96A and FAM96B induce breast cancer cell apoptosis}

Next, we detected the effects of FAM96A and FAM96B on breast cancer cell apoptosis. According to the flow cytometry analysis, cells were divided into four different groups including survival cells, early apoptotic cells, late apoptotic cells and necrotic cells. Here, we found that the percentage of apoptotic 
cells was increased, while the percentage of survival cells was decreased when FAM96A and FAM96B was overexpressed in breast cancer cells. Conversely, the percentage of apoptotic cells was decreased and the survival cells was increased when FAM96A and FAM96B was knockdowned in breast cancer cells (Fig. 4A, B). In order to investigate the molecular mechanism underlying the cell apoptosis induced by FAM96A and FAM96B, we then examined the expression changes of cell apoptosis-associated proteins including pro-apoptotic protein Bax, anti-apoptotic protein Bcl-2, and Caspase-3. Western blotting results showed that overexpression of FAM96A and FAM96B increased the expression of Bax and and proCaspase-3, but decreased the expression of Bcl-2. Moreover, we observed the significant cleavage of proCaspase-3 and the cleaved-Caspase 3 was increased in the FAM96A- and FAM96B-overexpressed groups compared to that in control group. Conversely, knockdown of FAM96A and FAM96B exhibited the opposite effects on the expression of apoptosis-associated proteins in both MCF-7 and MDA-MB-231 cells (Fig. 4C). Taken together, these data indicate that FAM96A and FAM96B induce breast cancer cell apoptosis through regulating cell death and survival protein expression.

\section{FAM96A and FAM96B suppress breast cancer cell migration and invasion}

We continued to investigate the functional roles of FAM96A and FAM96B in breast cancer cell migration and invasion abilities by wound healing and transwell assays. Wound healing analysis suggested that the scratch width in the FAM96A- and FAM96B-overexpressed cells was significantly larger than that of in the control cells, while knockdown of FAM96A and FAM96B promoted the wound healing of breast cancer cells compared to the corresponding siNC group (Fig. 5A, B). Consistent with the results of wound healing analysis, transwell cell migration and Matrigel invasion assays also showed that the amount of migrated and invaded cells remarkably decreased in FAM96A- and FAM96B-overexpressed groups, while the amount of migrated and invaded cells significantly increased in FAM96A- and FAM96B-depleted groups compared with the corresponding siNC group (Fig. 5C, D). Collectively, these results demonstrate that FAM96A and FAM96B suppress the invasion and migration abilities of breast cancer cells, which suggesting that FAM96A and FAM96B are probably tumor suppressor genes.

\section{FAM96A and FAM96B inhibit EMT of breast cancer cells}

It is also well known that cancer cell migration and invasion is closely related to epithelial-mesenchymal transition (EMT) [20]. To explore whether the suppressive migration and invasion effects on the breast cancer cells caused by FAM96A and FAM96B overexpression are associated with EMT, we analyzed the expression changes of EMT-associated markers using RT-qPCR and western blot assays. As shown in Fig. $6 \mathrm{~A}$ and $\mathrm{B}$, we found that overexpression of FAM96A and FAM96B in breast cancer cells increased the mRNA and protein levels of E-cadherin, but decreased the mRNA and protein expression levels of $\mathrm{N}$ cadherin, Vimentin, Snail and Slug. Consistently, knockdown of FAM96A and FAM96B in breast cancer cells led to the opposite effects on the mRNA and protein expression levels of EMT-associated markers. Taken together, these discoveries strongly prove that FAM96A and FAM96B suppress breast cancer cell invasion and migration abilities via inhibiting EMT. 
Many researches have shown that multiple signaling pathways are involved in regulating EMT process $[21,22]$. It has been well known that $W n t / \beta$-catenin signaling pathway is not only involved in the regulation of EMT, but also associated with tumorigenesis and metastasis [23]. To elucidate the specific molecular mechanisms by which FAM96A and FAM96B induced the inhibition of tumorigenesis and metastasis, we first explored the relationships between Wnt/ $\beta$-catenin signaling pathway and the biological functions of FAM96A and FAM96B in breast cancer. Our results showed that FAM96A and FAM96B overexpression resulted in the significant down-regulation of Wnt pathway effector $\beta$-catenin and Wnt pathway down-stream target genes cyclin D1 and c-Myc. In addition, FAM96A and FAM96B overexpression also caused the reduced phosphorylation level of GSK-3 $\beta$ (Ser 9), while total GSK-3 $\beta$ protein level showed no remarkable changes. On the contrary, knockdown of FAM96A and FAM96B resulted in the increased expression of $\beta$-catenin, cyclin D1 and c-Myc, as well as p-GSK-3 $\beta$, but the protein level of total GSK-3 $\beta$ still showed no visible changes in breast cancer cells (Fig. 7A, B).

Collectively, these data preliminarily indicate that overexpression of FAM96A and FAM96B may inhibit the activation of $\mathrm{Wnt} / \beta$-catenin signaling pathway in breast cancer.

To further confirm whether FAM96A and FAM96B suppressed the breast cancer progression through the inactivation of Wnt/ $\beta$-catenin signaling pathway, XAV-939, a specific Wnt/ $\beta$-catenin pathway inhibitor, was used to perform complementation experiments. As shown in Fig. 7C-E, the effects of FAM96A and FAM96B knockdown on promoting breast cancer cell proliferation, invasion and migration were also obviously weakened by XAV-939 treatment through MTT, transwell cell migration and Matrigel invasion assays. Moreover, XAV-939 treatment significantly attenuated the effects of FAM96A and FAM96B knockdown on the elevated protein expression of $\beta$-catenin, cyclin D1, c-Myc and p-GSK-3 $\beta$ (Fig. 7F). Furthermore, XAV-939 treatment reversed the suppressive effect of FAM96A and FAM96B knockdown on breast cancer cell apoptosis. Specifically speaking, XAV-939 treatment increased the protein level of Bax and decreased the protein level of Bcl-2 in the FAM96A- and FAM96B-depleted breast cancer cells compared to untreated group (Fig. 7G). Additionally, XAV-939 treatment inhibited the protein expression of EMT-associated markers including Vimentin, Snail and Slug compared with untreated group, which suggesting that XAV-939 treatment could attenuate the promoting effect of FAM96A and FAM96B knockdown on the EMT of breast cancer cells (Fig. 7G). Taken together, these results above all further confirm that FAM96A and FAM96B inhibit breast cancer cell proliferation, invasion and migration via the suppression of Wnt/ $\beta$-catenin signaling pathway. It is also indicated that both FAM96A and FAM96B may act as as tumor suppressor genes and play the important role in tumorigenesis and metastasis

\section{Discussion}

Although it has been found that FAM96A and FAM96B expression is down-regulated in some types of tumor tissues in comparison to the corresponding normal tissues, the functional roles and the molecular mechanisms of FAM96A and FAM96B in tumorigenesis and metastasis have not been fully elucidated, especially in the breast cancer progression. In this current research, we first observed that FAM96A and FAM96B expression was down-regulated in breast cancer. Functionally, our results showed that overexpression of FAM96A and FAM96B in breast cancer cells significantly inhibited the proliferation, 
invasion and migration, induced cell apoptosis and blocked cell cycle progression, while knockdown of FAM96A and FAM96B enhanced the malignant characteristics of breast cancer cells. Mechanistically, FAM96A and FAM96B inhibited breast cancer cell proliferation, invasion and migration via suppressing EMT and the activation of Wnt/ $\beta$-catenin signaling pathway, as illustrated in the schematic diagram in Fig. 8. Therefore, our data collectively support that FAM96A and FAM96B act as the tumor suppressor genes and participate in the tumorigenesis and metastasis.

Previous study has demonstrated that FAM96A is down-regulated in human gastrointestinal stromal tumors, and suppressed the tumorigenicity but induced apoptosis through direct interaction with Apaf-1. FAM96A is also considered as a new pro-apoptotic tumor suppressor in human gastrointestinal stromal tumors [14]. Another research work reported that the fusion protein of FAM96A and hTERT induced apoptosis, and inhibited proliferation, invasion and migration of liver cancer cells [16]. However, there are few reports on the role of FAM96B in tumor progression. FAM96B is only known to have an inhibitory role in Apoptin-induced apoptosis through interaction with Apoptin. In the present study, we found that both FAM96A and FAM96B overexpression in breast cancer cells inhibited cell proliferation and induced cell apoptosis. Meanwhile, FAM96A and FAM96B overexpression resulted in the down-regulation of Bcl-2 expression and up-regulation of Bax and cleaved-Caspase 3 expression. Conversely, FAM96A and FAM96B knockdown exerted the opposite effects on breast cancer cell proliferation and apoptosis. Our data further indicate that FAM96A and FAM96B may play the pro-apoptotic role in the mitochondrial apoptosis pathway.

Invasion and metastasis are the main characteristics of malignant tumors, and also the main reasons of high mortality in breast cancer patients [24]. According to some previous domestic studies, the mRNA expression levels of FAM96A and FAM96B were down-regulated in colon cancer, liver cancer and gastric cancer. Overexpression of FAM96A and FAM96B in these kinds of cancer cells inhibited the cell proliferation and induced cell apoptosis, but the functional role of FAM96A and FAM96B in the tumorigenesis and metastasis was not clear. Here, we first found that FAM96A and FAM96B overexpression significantly suppressed the breast cancer cell invasion and migration capabilities, while FAM96A and FAM96B knockdown enhanced the invasion and migration capabilities, which indicated that FAM96A and FAM96B played the tumor suppressive roles in the breast cancer progression.

It has been well established that EMT plays an important role in the process of tumor invasion and metastasis [25]. More importantly, our present results showed that FAM96A and FAM96B were also involved in the regulation of EMT. Overexpression of FAM96A and FAM96B inhibited EMT by upregulating the mRNA and protein expression of the E-cadherin, and down-regulating the mRNA and protein levels of N-cadherin, Vimentin, Slug and Snail in breast cancer cells. Conversely, FAM96A and FAM96B knockdown caused the opposite effects on the expression changes of EMT-associated markers. Therefore, our findings suggest for the first time that FAM96A and FAM96B are indeed associated with EMT, and both of them suppress the invasion and migration of breast cancer cells via the inhibition of EMT. 
EMT has been known to be associated with the abnormal activation or inactivation of Wnt/ $\beta$-catenin signaling pathway, which is involved in the tumor growth and metastasis [25]. Here, our data displayed that overexpression of FAM96A and FAM96B led to the reduction of the phosphorylation level of GSK-3 $\beta$ and decrease the protein expression of $\beta$-catenin, as well as the down-stream target genes of Wnt pathway including cyclin D1 and c-Myc, which suggested that FAM96A and FAM96B overexpression results in the inactivation of Wnt/ $\beta$-catenin signaling pathway in breast cancer. Additionally, FAM96A and FAM96B knockdown resulted in the opposite effects on the expression changes of above these proteins. In order to further confirm our conclusions, we used a specific Wnt/ $\beta$-catenin inhibitor XAV-939 to block Wnt pathway, and found that XAV-939 treatment obviously attenuated the effects of FAM96A and FAM96B knockdown on the elevated expression of p-GSK-3 $\beta, \beta$-catenin, cyclin D1 and c-Myc. In addition, the effects of FAM96A and FAM96B knockdown on promoting the proliferation, invasion, migration and EMT of breast cancer cells were also reversed by XAV-939 treatment. Therefore, our results provide first evidence for that FAM96A and FAM96B overexpression inhibit breast cancer cell proliferation, invasion and migration abilities via suppressing the $W n t / \beta$-catenin signaling pathway. For the first time, we identify that FAM96A and FAM96B may serve as the novel tumor suppressor genes and potential therapeutic targets for breast cancer. We propose that further investigations towards in vivo observations with FAM96A and FAM96B are needed to elucidate the underlying molecular mechanisms in the tumor progression.

\section{Conclusion}

In conclusions, our results provide first evidence for that FAM96A and FAM96B expression is downregulated in breast cancer. Overexpression of FAM96A and FAM96B inhibit breast cancer cell proliferation, invasion and migration abilities through the suppression of Wnt/ $\beta$-catenin signaling pathway. Our studies expand the current outstanding of FAM96A and FAM96B in cancer biology. These findings highlight the importance of FAM96A and FAM96B as tumor suppressor genes in breast cancer progression, and further verify that FAM96A and FAM96B may be the novel diagnostic markers and the potential therapeutic targets for breast cancer.

\section{Abbreviations}

FAM96A, family with sequence similarity 96 member A; FAM96B, family with sequence similarity 96 member B; DMEM, Dulbecco's modified Eagle's medium; PBS, phosphate-buffered saline; FBS, fetal bovine serum; PI, propidium iodide; MTT, 3-(4,5-Dimethylthiazol-2-yl)-2,5-diphenyltetrazolium bromide; RTqPCR, quantitative reverse transcription-PCR; siRNA, small interfering RNA; EMT, epithelial-mesenchymal transition; CIA01, cytosolic iron-sulfur assembly component 1; DUF59, domain of unknown function 59; Apaf-1, apoptotic protease activating factor-1; IRP1, iron regulatory protein 1; IRP2, iron regulatory protein 2; ANT2, ADP/ATP transposase 2; SelW, selenoprotein W; hTERT, human telomerase reverse transcriptase; hTERTR, human telomerase reverse transcriptase receptor. 


\section{Declarations}

Acknowledgements

Not applicable.

\section{Authors' contributions}

LNZ and DDZ made the substantial contributions to the study conception. LNZ conceived and designed the study. LNZ and DDZ performed the experiments and analyzed the data. XLS participated in the western blot analysis. ZYL helped to complete the flow cytometry analysis. LNZ drafted and revised the manuscript. All authors have read and approved the published version of the final manuscript.

\section{Funding}

The present study was supported by the National Natural Science Foundation of China (Grant No. 82172969, 81702272), Beijing Natural Science Foundation (Grant No. 5202001), and 2021 Talent Training Innovation Foundation from Faculty of Environment and Life of Beijing University of Technology.

\section{Availability of data and materials}

The datasets used and analyzed during the present study are available from the corresponding author on reasonable request.

\section{Ethics approval and consent to participate}

Not applicable.

\section{Consent for publication}

Not applicable.

\section{Competing interests}

The authors declare that they have no competing interests.

\section{References}

1. DeSantis CE, Ma J, Gaudet MM, Newman LA, Miller KD, Goding Sauer A, et al. Breast cancer statistics, 2019. CA Cancer J Clin. 2019, 69(6): 438-451.

2. Chen KE, Richards AA, Ariffin JK, Ross IL, Sweet MJ, Kellie S, et al. The mammalian DUF59 protein Fam96a forms two distinct types of domain-swapped dimer. Acta Crystallogr D Biol Crystallogr. 2012, 68(Pt 6): 637-648. 
3. Balk J, Aguilar Netz DJ, Tepper K, Tepper K, Pierik AJ, Lill R. The essential WD40 protein Cia1 is involved in a late step of cytosolic and nuclear iron-sulfur protein assembly. Mol Cell Biol. 2005, 25(24): 10833-10841.

4. Stehling O, Mascarenhas J, Vashisht AA, Sheftel AD, Niggemeyer B, Rosser R, et al. Human CIA2AFAM96A and CIA2B-FAM96B integrate iron homeostasis and maturation of different subsets of cytosolic-nuclear iron-sulfur proteins. Cell Metab. 2013, 18(2): 187-198.

5. Maione V, Cantini F, Severi M, Banci L. Investigating the role of the human CIA2A-CIAO1 complex in the maturation of aconitase. Biochim Biophys Acta-Gen Subj. 2018, 1862(9): 1980-1987.

6. van Wietmarschen N, Moradian A, Morin GB, Lansdorp PM, Uringa EJ. The mammalian proteins MMS19, MIP18, and ANT2 are involved in cytoplasmic iron-sulfur cluster protein assembly. J Biol Chem. 2012, 287(52): 43351-43358.

7. Ito S, Tan LJ, Andoh D, Narita T, Seki M, Hirano Y, et al. MMXD, a TFIIH-independent XPD-MMS19 protein complex involved in chromosome segregation. Mol Cell. 2010, 39(4): 632-640.

8. Yin A, Luo Y, Chen W, He M, Deng JH, Zhao N, et al. FAM96A Protects Mice From Dextran Sulfate Sodium (DSS)-Induced Colitis by Preventing Microbial Dysbiosis. Front Cell Infect Mi. 2019, 9: 381.

9. Yin A, Chen W, Cao L, Li Q, Zhu X, Wang L. FAM96A knock-out promotes alternative macrophage polarization and protects mice against sepsis. Clin Exp Immunol. 2021, 203(3): 433-447.

10. Yang W, Itoh F, Ohya H, Kishimoto F, Tanaka A, Nakano N, et al. Interference of E2-2-mediated effect in endothelial cells by FAM96B through its limited expression of E2-2. Cancer Sci. 2011, 102(10): 1808-1814.

11. Chen P, Wang C, Liu Q, Tian J, Liu Q. Identification of FAM96B as a novel selenoprotein W binding partner in the brain. Biochem Biophys Res Commun. 2019, 512(1): 137-143.

12. Xiong XD, Wang J, Zheng H, Jing X, Liu Z, Zhou Z, et al. Identification of FAM96B as a novel prelamin A binding partner. Biochem Biophys Res Commun. 2013, 440(1): 20-24.

13. Liang H, Li W, Yang H, Cao Y, Ge L, Shi R, et al. FAM96B inhibits the senescence of dental pulp stem cells. Cell Bio Int. 2020, 44(5): 1193-1203.

14. Schwamb B, Pick R, Fernandez SB, Volp K, Heering J, Dotsch V, et al. FAM96A is a novel proapoptotic tumor suppressor in gastrointestinal stromal tumors. Int J Cancer. 2015, 137(6): 13181329.

15. Danen-Van Oorschot AA, Zhang YH, Leliveld SR, Rohn JL, Seelen MC, Bolk MW, et al. Importance of nuclear localization of apoptin for tumor-specific induction of apoptosis. J Biol Chem. 2003, 278(30): 27729-27736.

16. Zhang MY, Wang JP. A multi-target protein of hTERTR-FAM96A presents significant anticancer potent in the treatment of hepatocellular carcinoma. Tumour Biol. 2017, 39(4): 1010428317698341.

17. Wang WP, Gao HY. Combination therapy of hTERTR and FAM96A for hepatocellular carcinoma through enhancing apoptosis sensitivity. Exp Ther Med. 2018, 15(1): 641-648. 
18. Rhodes DR, Kalyana-Sundaram S, Mahavisno V, Varambally R, Yu J, Briggs BB, et al. Oncomine 3.0: genes, pathways, and networks in a collection of 18,000 cancer gene expression profiles. Neoplasia. 2007, 9(2): 166-180.

19. Besson A, Dowdy SF, Roberts JM. CDK inhibitors: cell cycle regulators and beyond. Dev Cell. 2008, 14(2): 159-169.

20. Nieto MA, Huang RY, Jackson RA, Thiery JP. Emt: 2016. Cell. 2016, 166(1): 21-45.

21. Lamouille S, Xu J, Derynck R. Molecular mechanisms of epithelial-mesenchymal transition. Nat Rev Mol Cell Biol. 2014, 15(3): 178-196.

22. Zhang J, Tian XJ, Xing J. Signal Transduction Pathways of EMT Induced by TGF-beta, SHH, and WNT and Their Crosstalks. J Clin Med. 2016, 5(4): 41.

23. Zhan T, Rindtorff N, Boutros M. Wnt signaling in cancer. Oncogene. 2017, 36(11): 1461-1473.

24. Leber MF, Efferth T. Molecular principles of cancer invasion and metastasis (review). Int J Oncol. 2009, 34(4): 881-895.

25. Heerboth S, Housman G, Leary M, Longacre M, Byler S, Lapinska K, et al. EMT and tumor metastasis. Clin Transl Med. 2015, 4: 6.

\section{Figures}


A

FAM96A

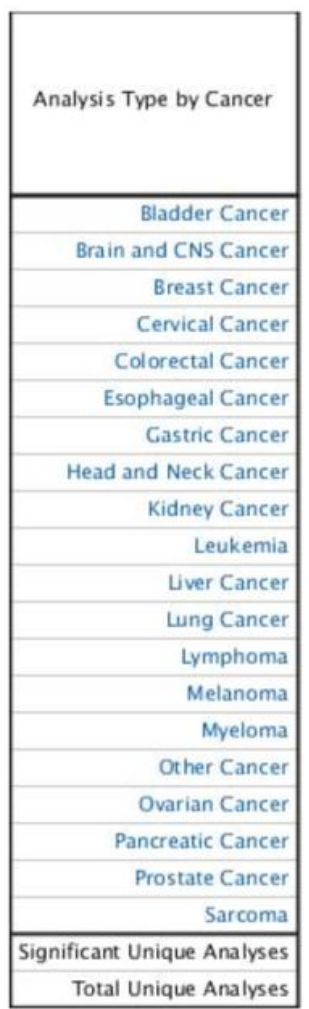

C

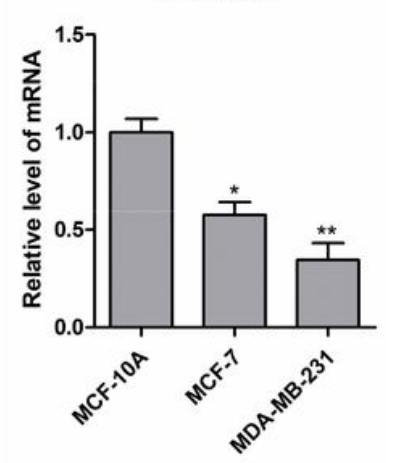

FAM96B

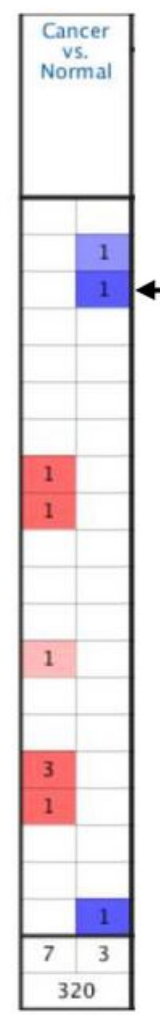

Bain and CNS Car
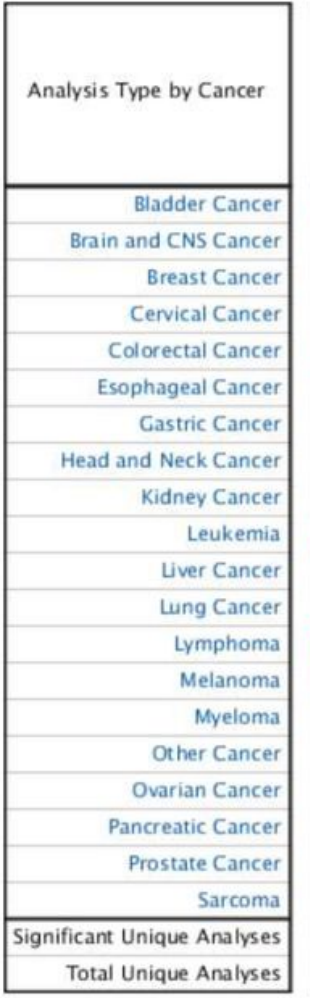

FAM96B

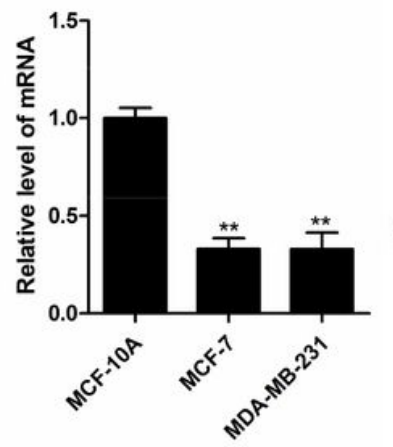

B

FAM96A Expression in Finak Breast Invasive Breast Carcinoma vs. Normal
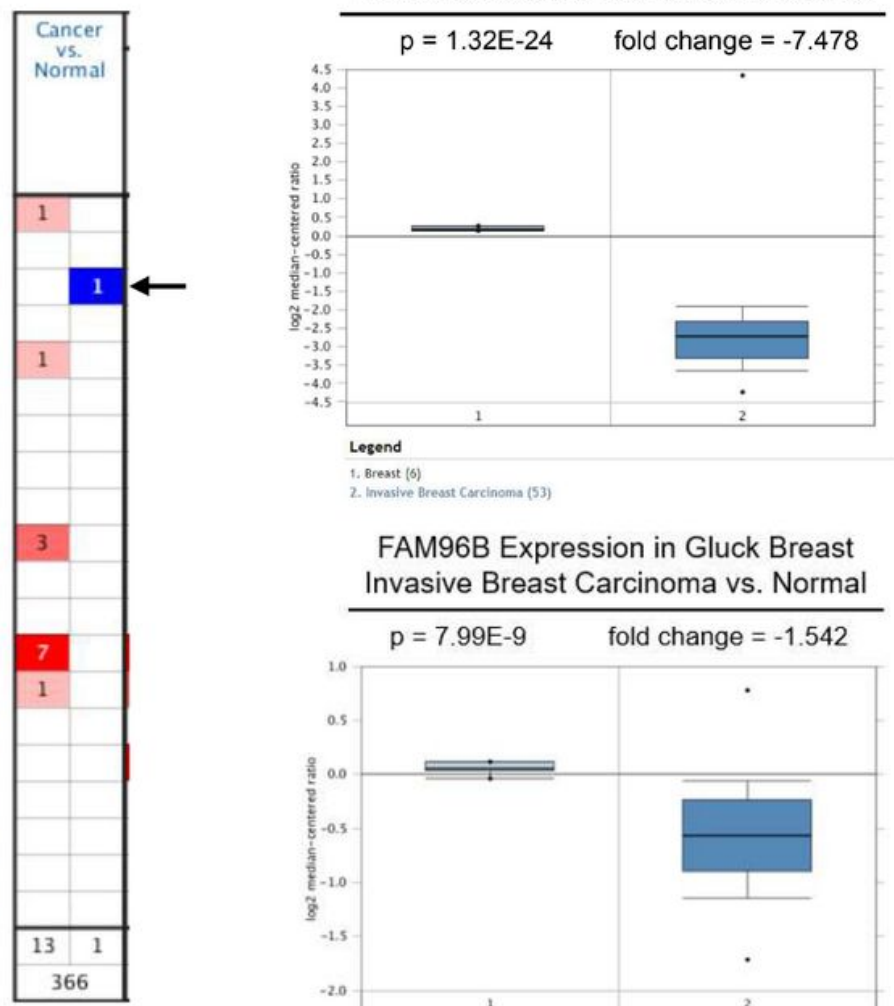

FAM96B Expression in Gluck Breast Invasive Breast Carcinoma vs. Normal

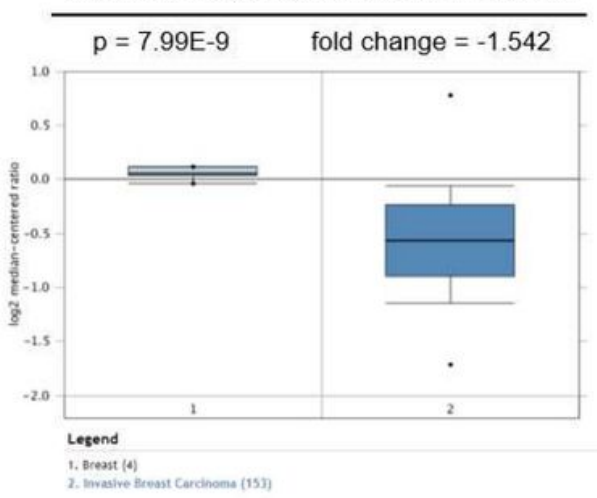

D
FAM96A

$\beta$-actin

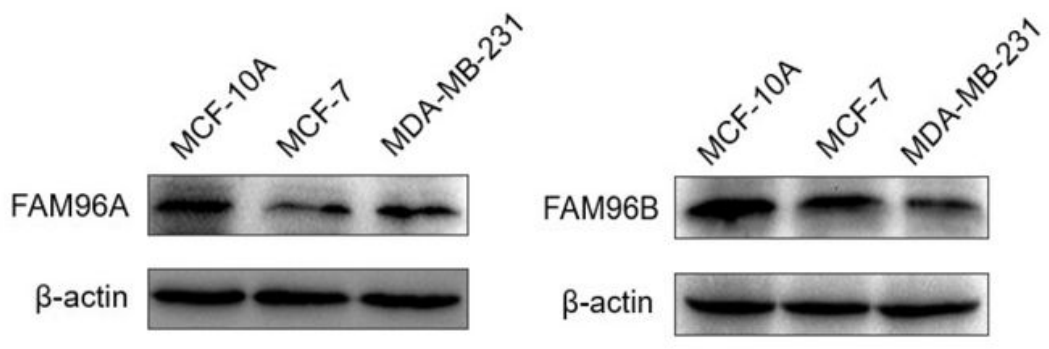

\section{Figure 1}

FAM96A and FAM96B expression is down-regulated in breast cancer. (A) Changes in the mRNA levels of FAM96A and FAM96B in different types of cancer analyzed by Oncomine database. (B) Changes in the mRNA levels of FAM96A and FAM96B between the invasive breast carcinoma and normal breast tissue of a specific breast cancer dataset from Oncomine database were analyzed. (C) The mRNA expression levels of FAM96A and FAM96B in the two breast cancer cell lines MCF-7 and MDA-MB-231 and normal breast epithelial cell line MCF-10A were determined by RT-qPCR. The relative mRNA expression levels were qualified by the abundance of GAPDH mRNA and expressed as mean \pm SEM of three independent experiments. (D) The protein expression levels of FAM96A and FAM96B in MCF-10A, MCF-7 and MDAMB-231 cells were examined by Western blot. $\beta$-actin was used as an internal loading control. ${ }^{*} p<0.05$, ${ }^{*} \mathrm{p}<0.01$. 

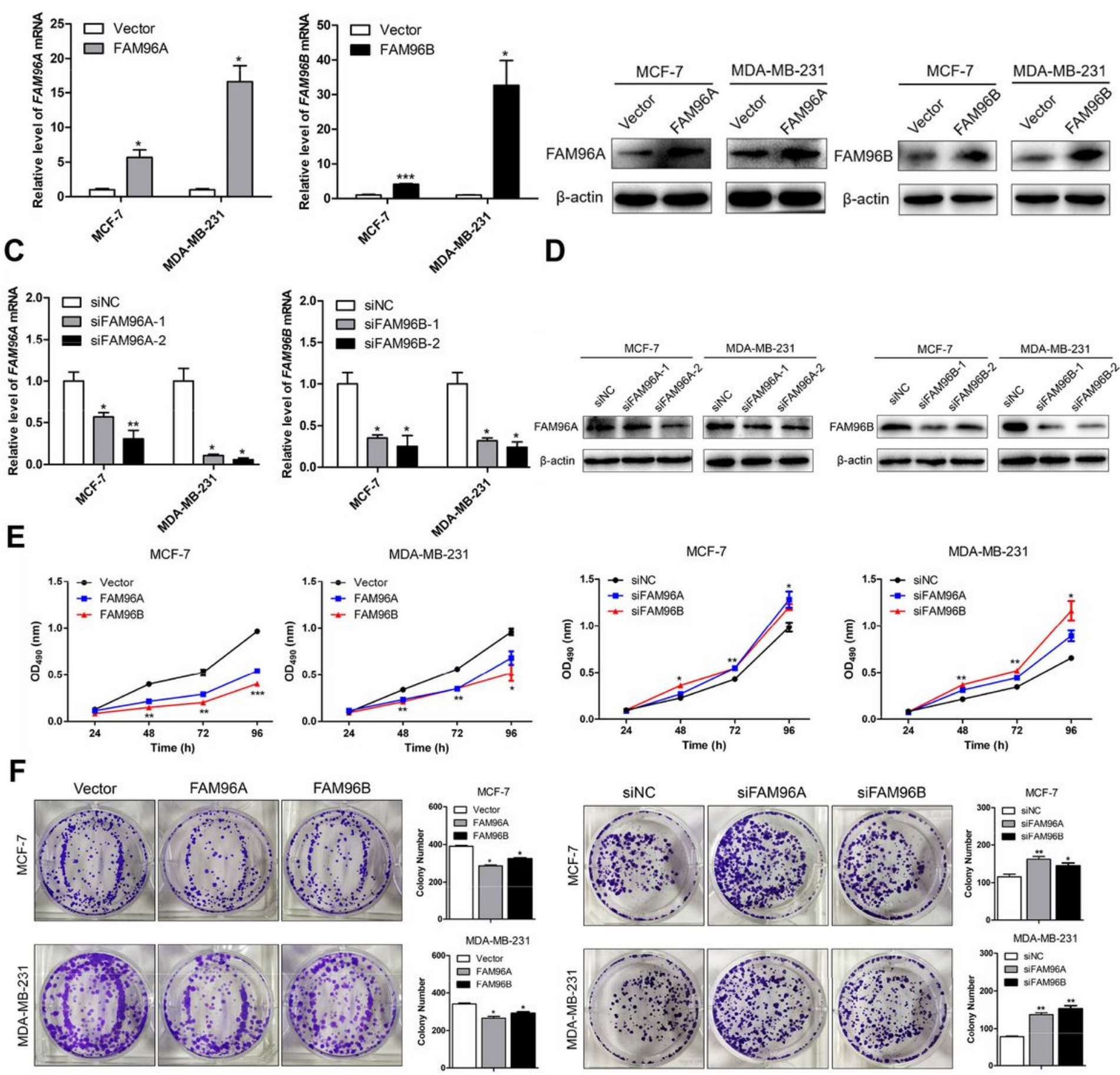

Figure 2

FAM96A and FAM96B inhibit the proliferation abilities of breast cancer cells. (A, B) The overexpression efficiencies of FAM96A and FAM96B in MCF-7 and MDA-MB-231 cells were confirmed by RT-qPCR and Western blot assays. (C, D) The knockdown efficiencies of FAM96A and FAM96B in MCF-7 and MDA-MB231 cells were determined by RT-qPCR and Western blot assays. (E) Cell proliferation rates of MCF-7 and MDA-MB-231 cells were detected by MTT assay after FAM96A and FAM96B overexpression or knockdown. (F) Colon formation abilities of MCF-7 and MDA-MB-231 cells were assayed after FAM96A 
and FAM96B overexpression or knockdown. Vector, cells were transfected with empty vector, FAM96A and FAM96B, cells were transfected with vector expressing FAM96A and FAM96B, respectively. NC, negative control, siFAM96A and siFAM96B, cells were transfected with siRNA targeting for FAM96A and FAM96B, respectively. All values are exhibited as mean \pm SEM of three independent experiments. ${ }^{*} p<0.05,{ }^{*} p<0.01$, and $* * * p<0.001$.

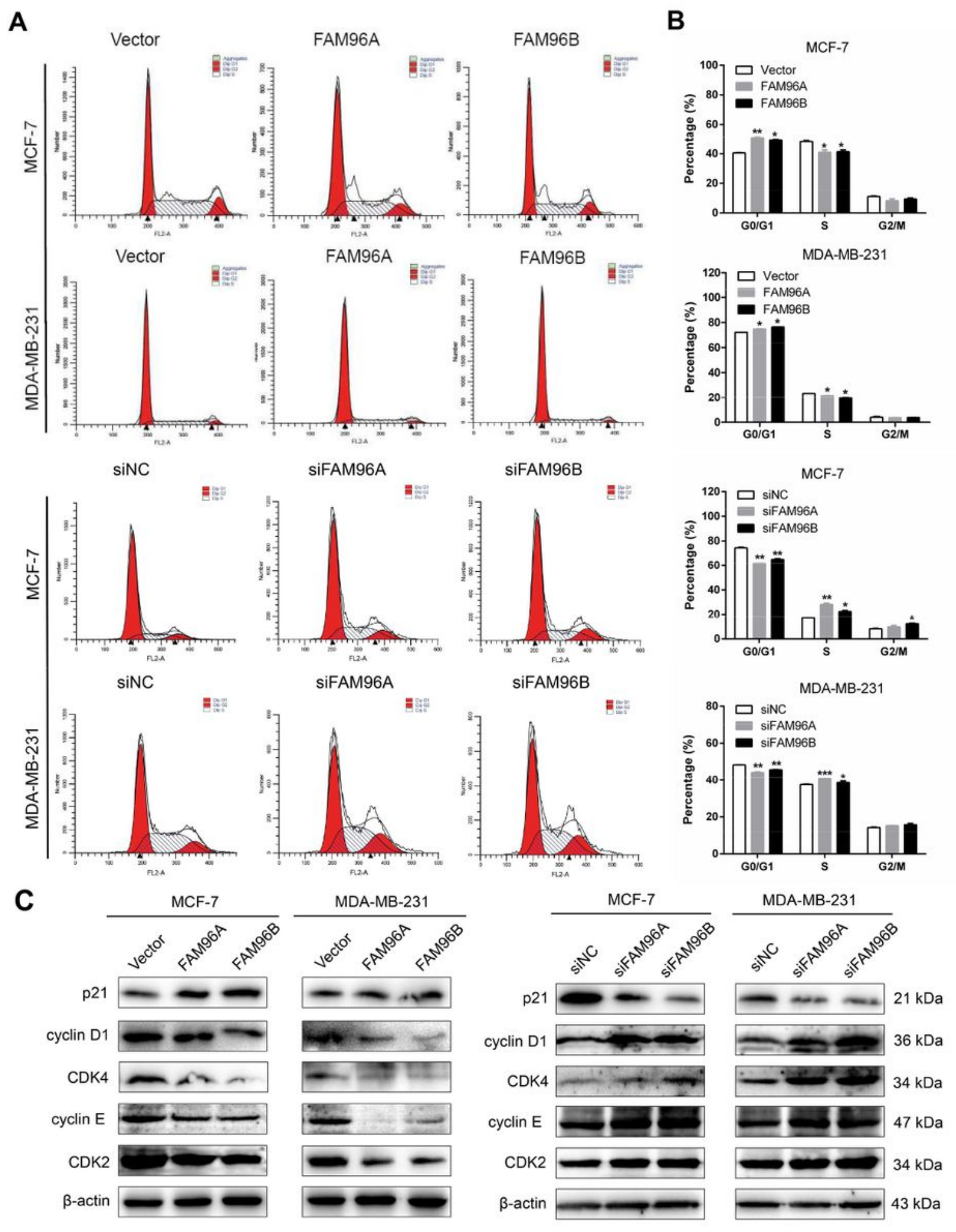

Figure 3 
FAM96A and FAM96B restard breast cancer cell cycle progression. (A) Representative images of breast cancer cell cycle examined by PI staining and flow cytometry. (B) The proportions of breast cancer cells in the $G 0 / G 1, S$ and $G 2 / M$ phases quantified from the flow cytometry analysis of 20,000 cells after FAM96A and FAM96B overexpression or knockdown. (C) Effects of FAM96A and FAM96B overexpression or knockdown on the protein levels of cell cycle-associated proteins including p21, CDK4, cyclin D1, CDK2 and cyclin E. $\beta$-actin was served as the protein loading control. ${ }^{*} p<0.05,{ }^{* *} p<0.01$, and ${ }^{* * *} p<0.001$.

A
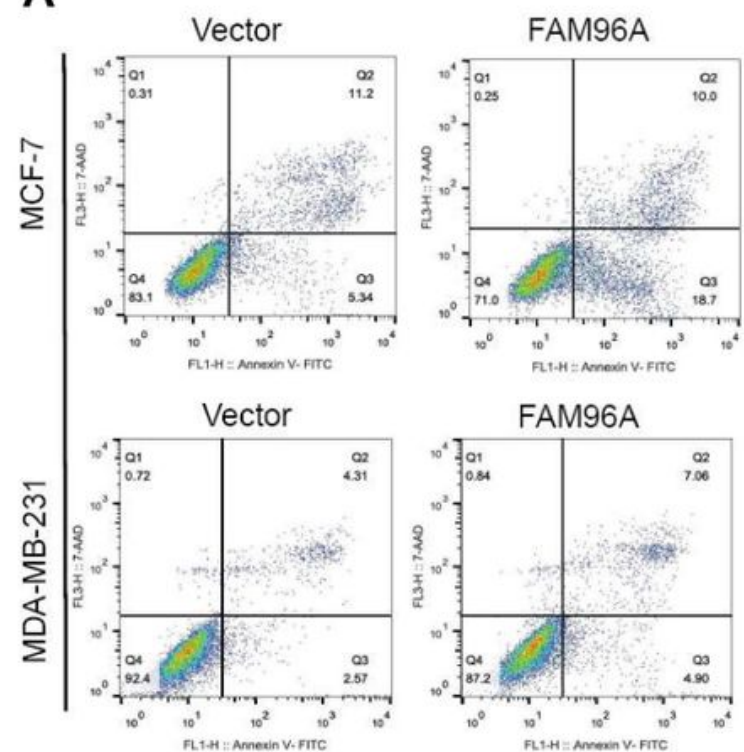

siNC
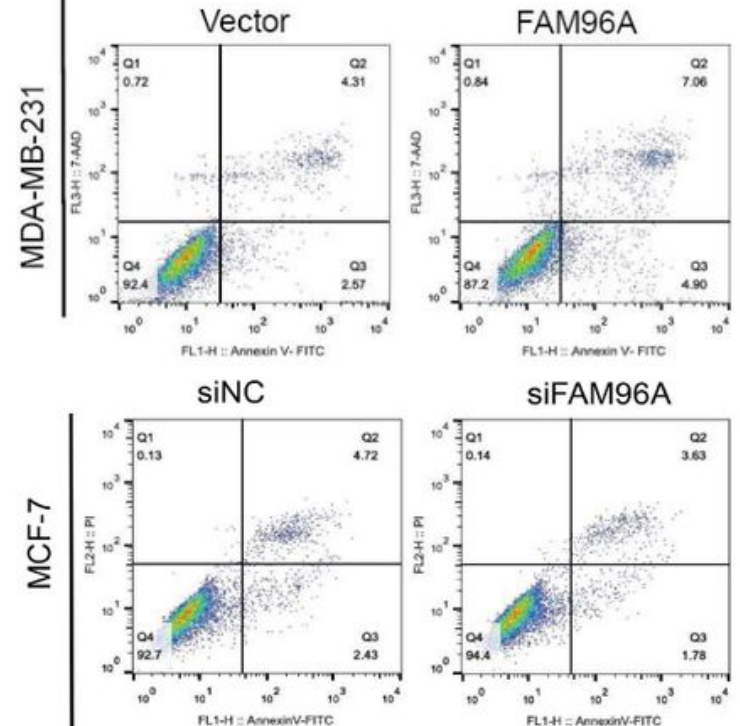

SiFAM96A
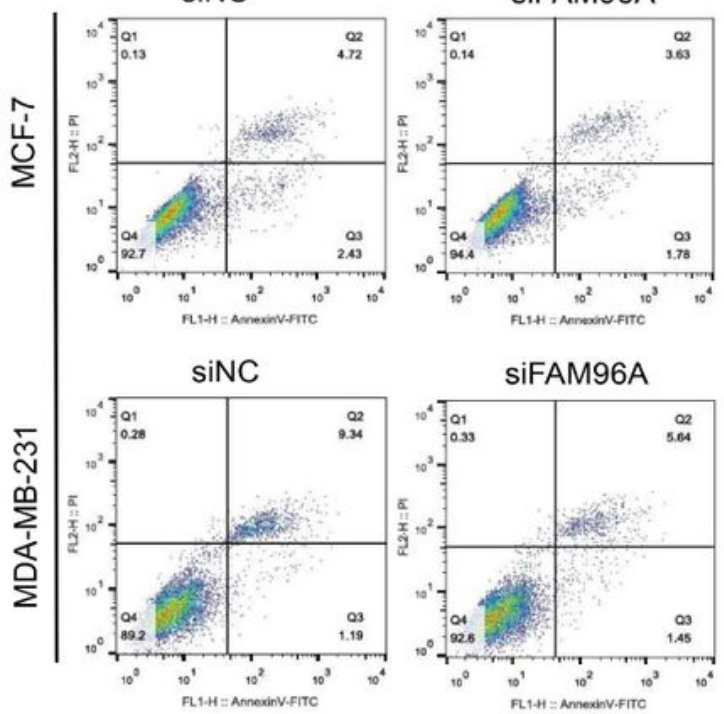

C

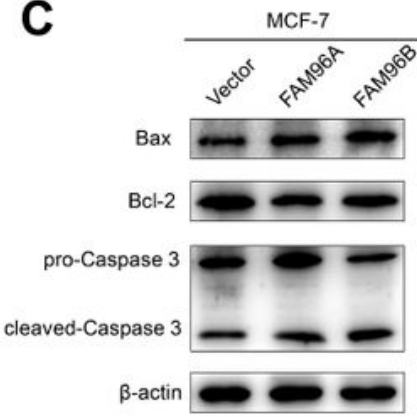

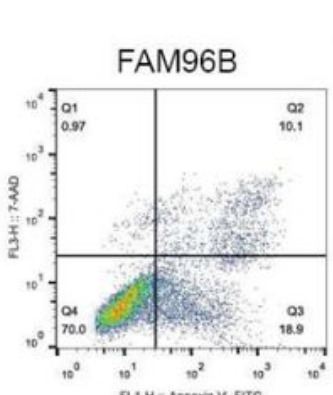

FAM96B
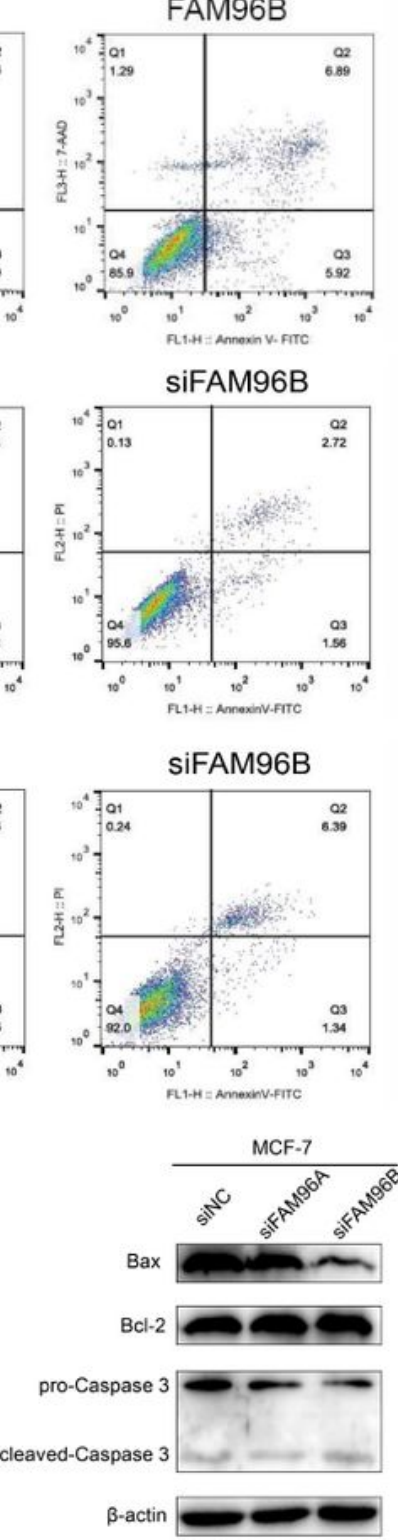

B

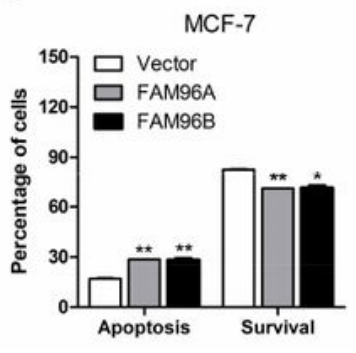

MDA-MB-231
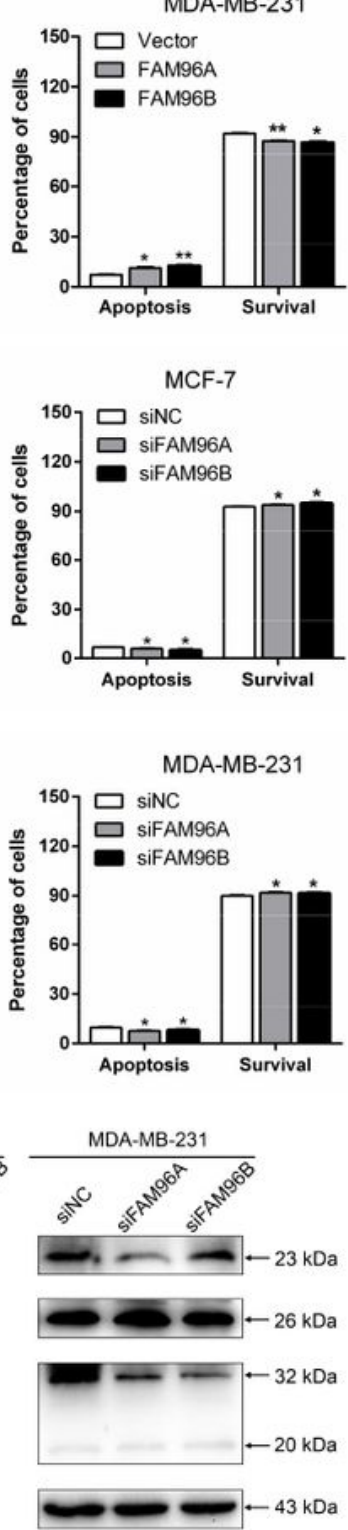
FAM96A and FAM96B induce breast cancer cell apoptosis. (A) Representative images of breast cancer cell apoptosis examined by Annexin V-FITC/PI or Annexin V-FITC/7-AAD apoptosis kit staining and flow cytometry. (B) The proportions of apoptotic cells and survival cells were qualified from Annexin V-positive populations and Annexin V-negative populations, respectively, after FAM96A and FAM96B overexpression or knockdown. Results were displayed as mean \pm SEM in three independent experiments. (C) Cell apoptosis-associated proteins including Bcl-2, Bax and Caspase 3 were detected by Western blotting after FAM96A and FAM96B overexpression or knockdown. $\beta$-actin was used as an internal control for protein loading. These data are the representative of at least three independent experiments. ${ }^{*} p<0.05,{ }^{* *} p<0.01$. 
A
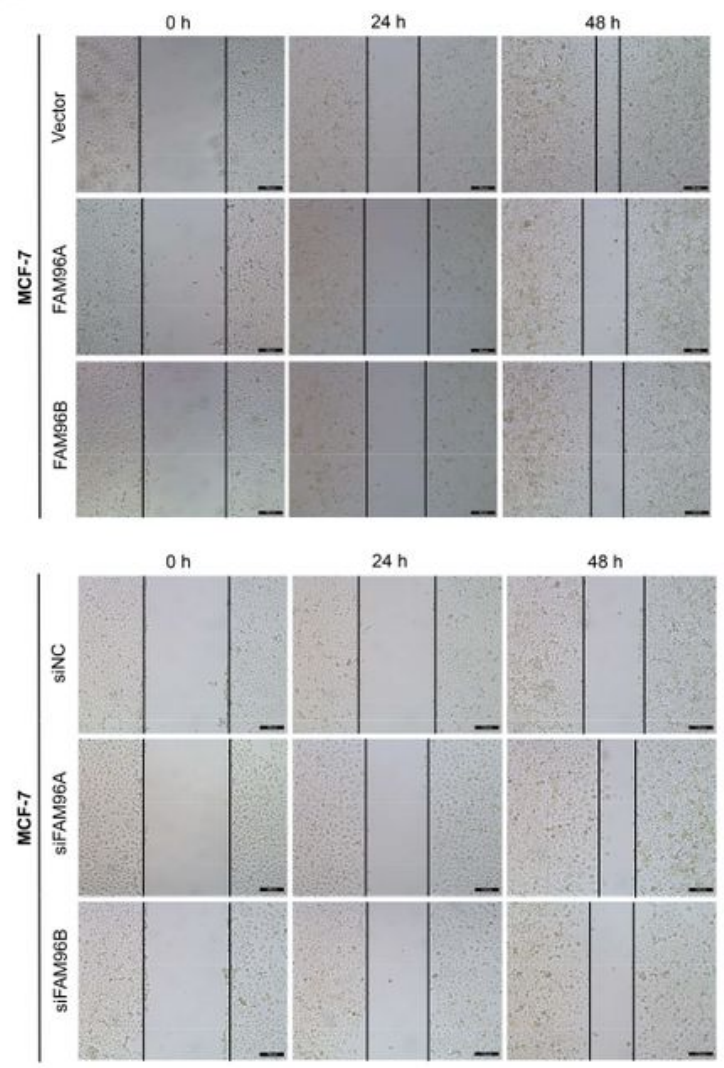

C

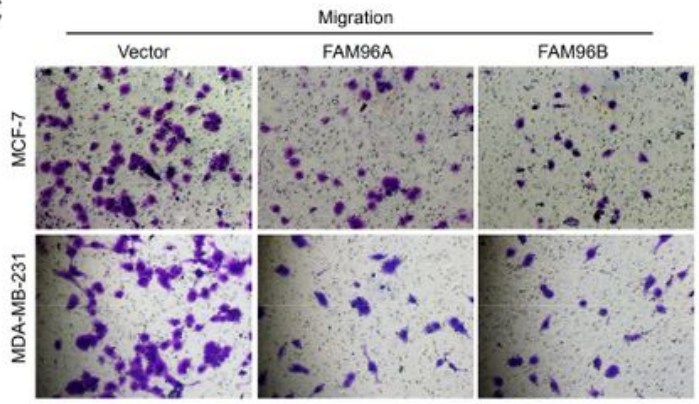

D

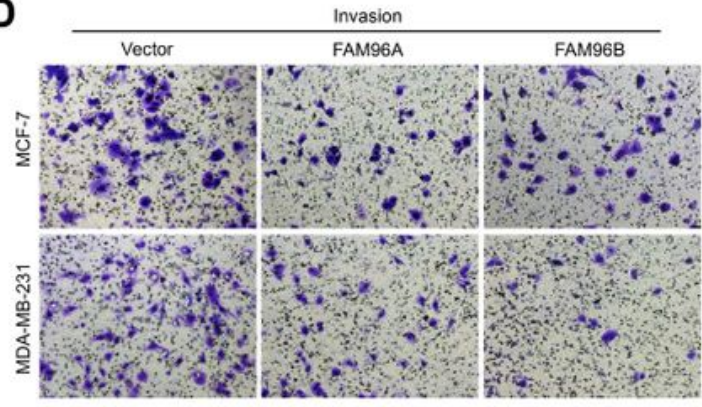

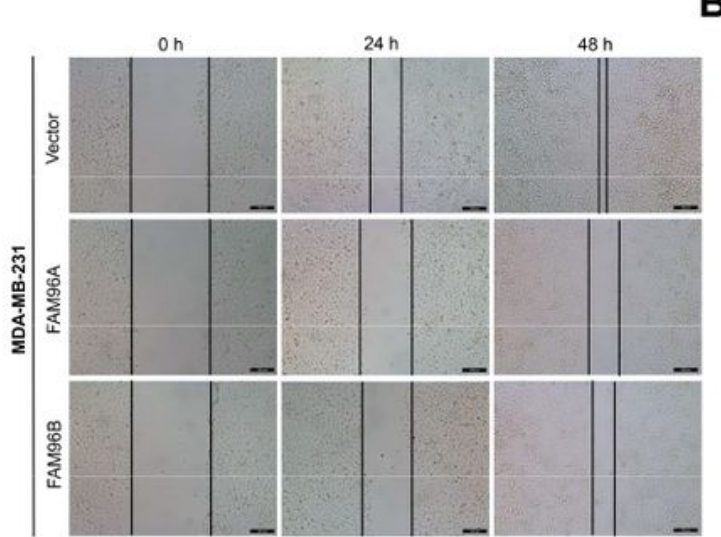

B
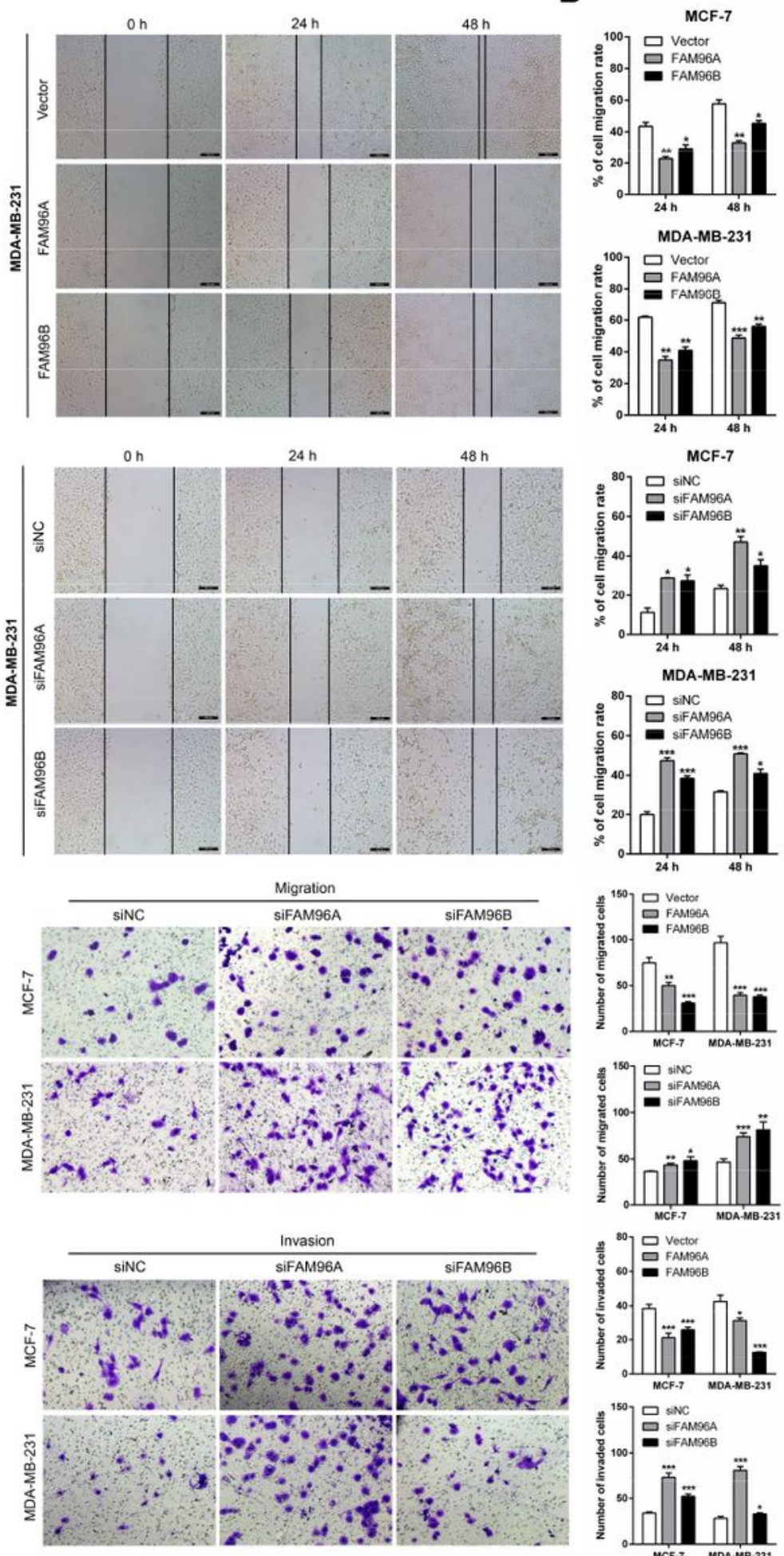
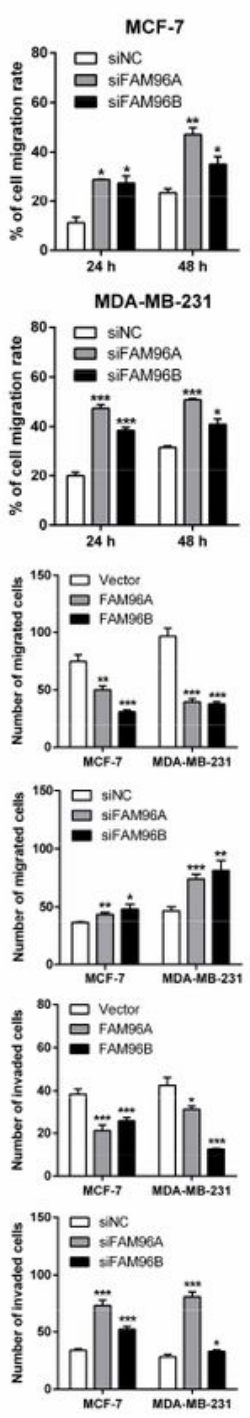

Figure 5

FAM96A and FAM96B suppress breast cancer cell migration and invasion abilities. (A) Effects of FAM96A and FAM96B overexpression or knockdown on the migration of breast cancer cells determined by wound healing assay. Scare bars represent $100 \mu \mathrm{m}$. The exhibited data were a representative of three independent experiments. (B) The relative migration rate of breast cancer cells calculated after FAM96A and FAM96B overexpression or knockdown. (C) Effects of FAM96A and FAM96B overexpression or 
knockdown on the migration ability of breast cancer cells detected using transwell cell migration assay. (D) Effects of FAM96A and FAM96B overexpression or knockdown on the invasion ability of breast cancer cells measured with transwell Matrigel invasion assay. The number of migrated and invaded cells were counted in ten randomly selected microscopic fields. Relative quantification data were the mean \pm SEM of three independent experiments. ${ }^{*} p<0.05,{ }^{* *} p<0.01$, and ${ }^{* * *} p<0.001$.

A

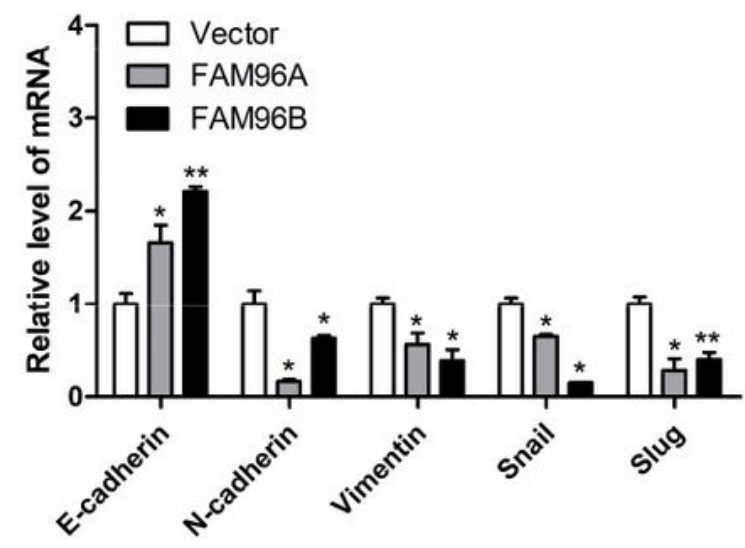

MCF-7

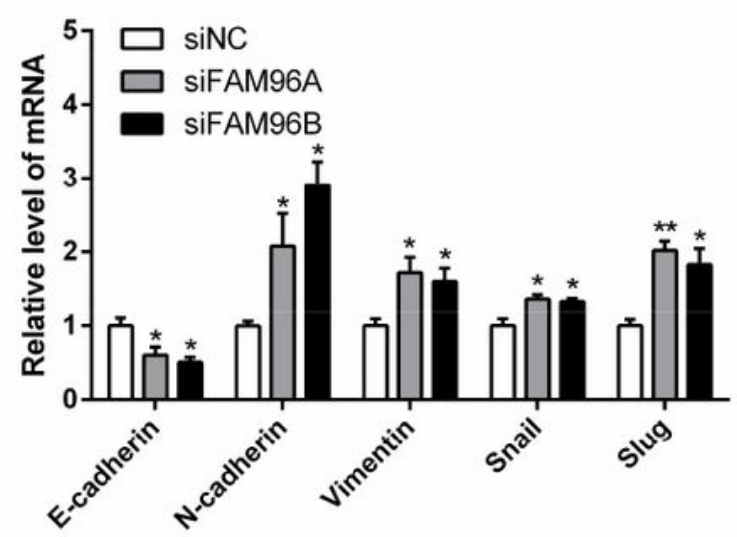

B

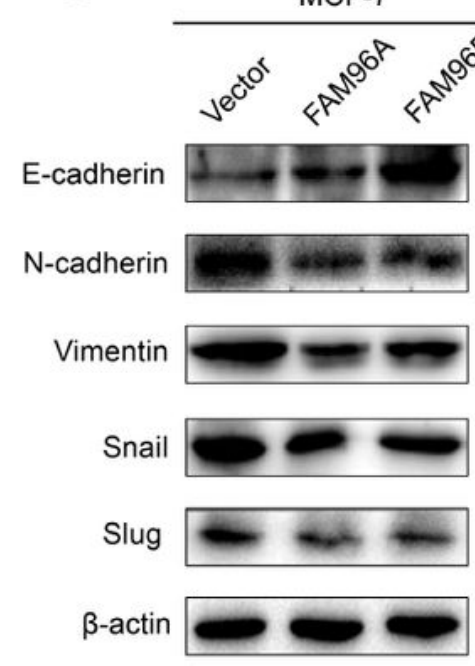

MCF-7

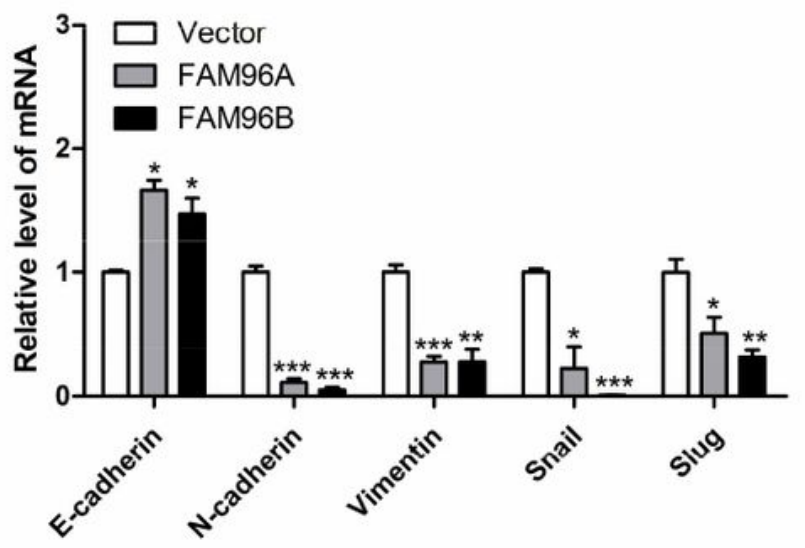

MDA-MB-231
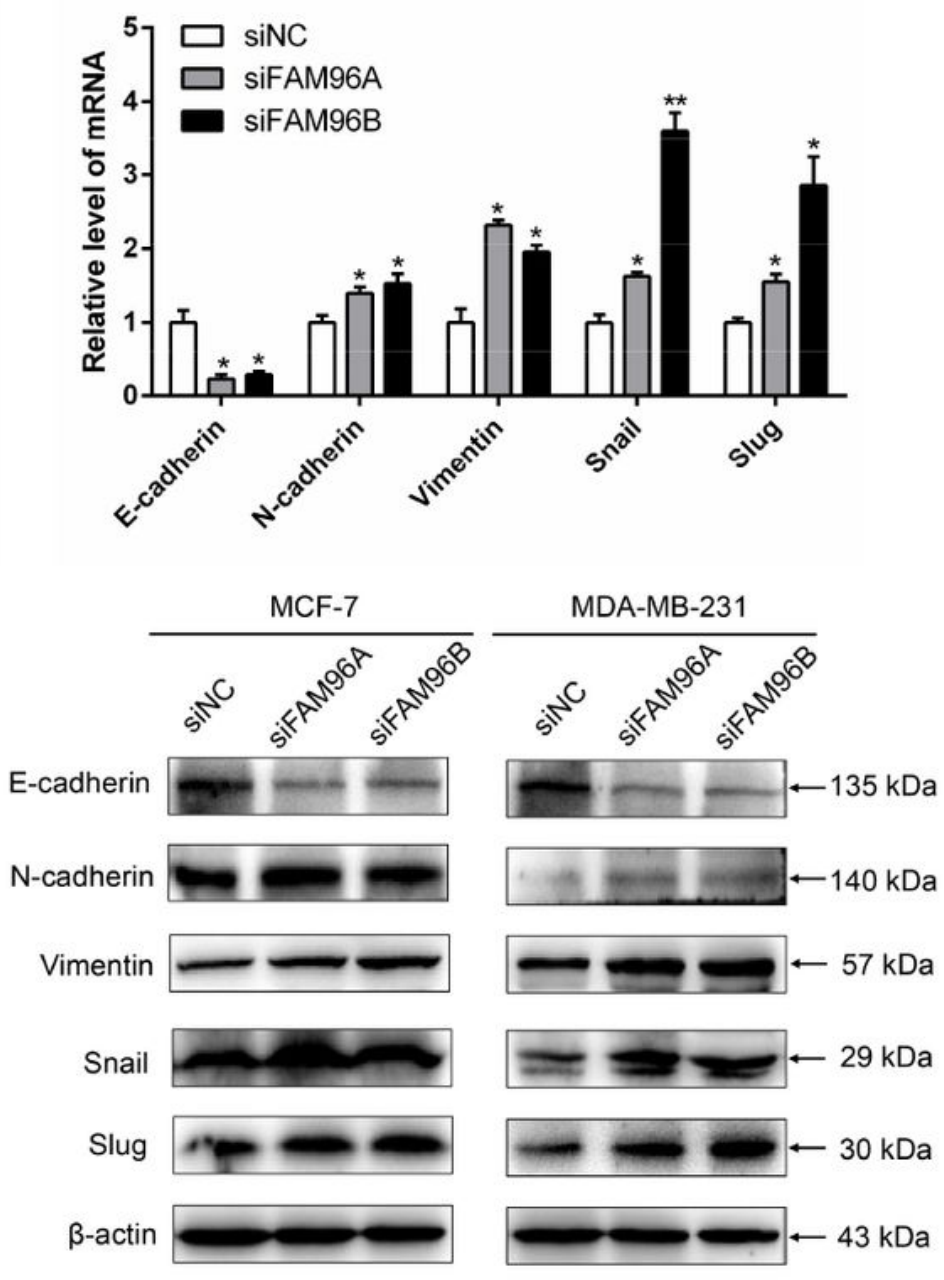

Figure 6 
FAM96A and FAM96B inhibit EMT of breast cancer cells. (A) Changes in the mRNA levels of EMTassociated markers including E-cadherin, N-cadherin, Vimentin, Snail and Slug analyzed by RT-qPCR after FAM96A and FAM96B overexpression or knockdown. (B) Expression changes in the protein levels of EMTassociated markers assessed by western blotting after FAM96A and FAM96B overexpression or knockdown in breast cancer cells. ${ }^{*} p<0.05,{ }^{* *} p<0.01$, and ${ }^{* * *} p<0.001$.

A
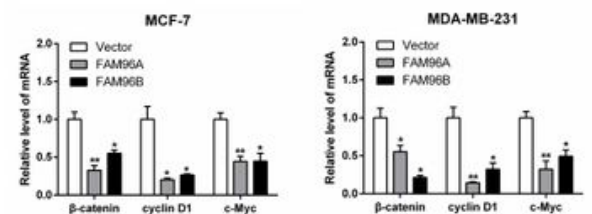

B
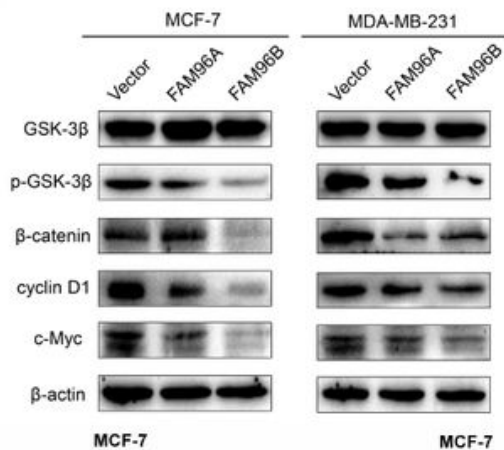

C
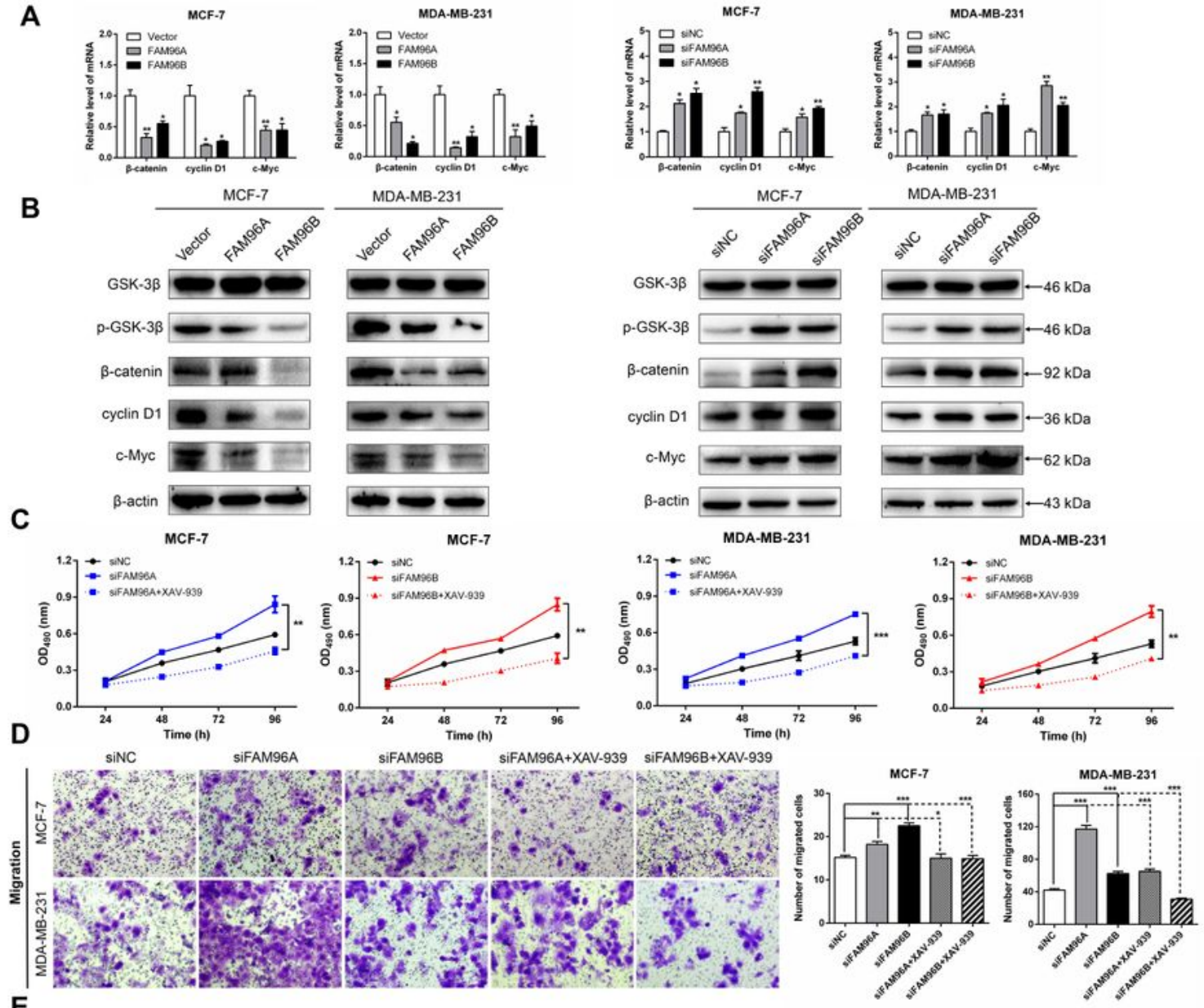

$\mathrm{E}$
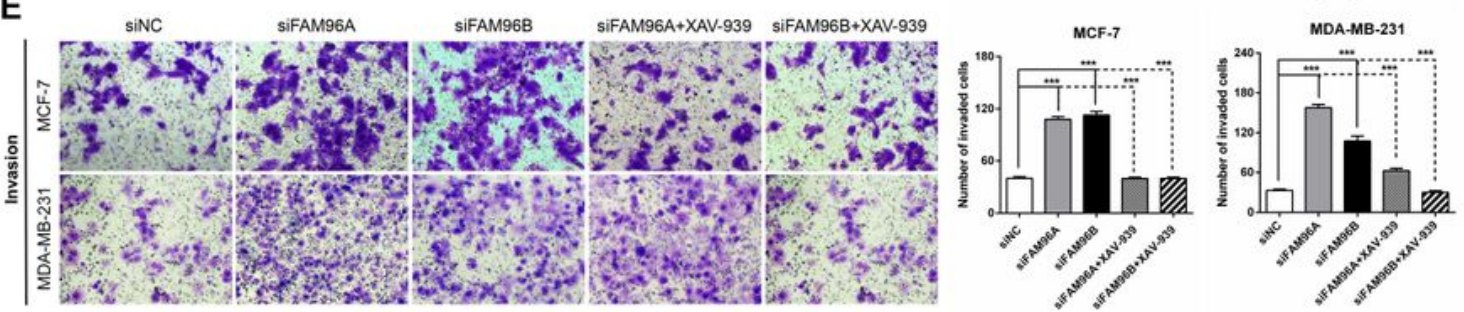

$\mathbf{F}$

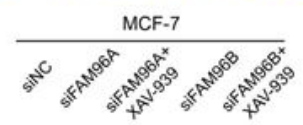

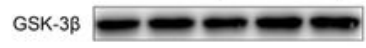

p-GSK-3B $-0--$

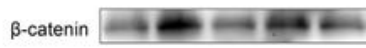

cyclin $\mathrm{D} 1 \cdots 0 \cdots$

c.Myc $\mathrm{B}$ का

B-actin $-\longrightarrow$
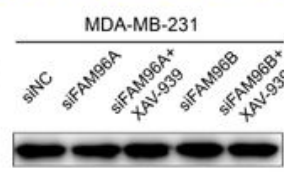

$-0-m-$

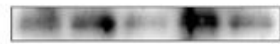
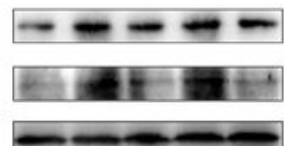

G
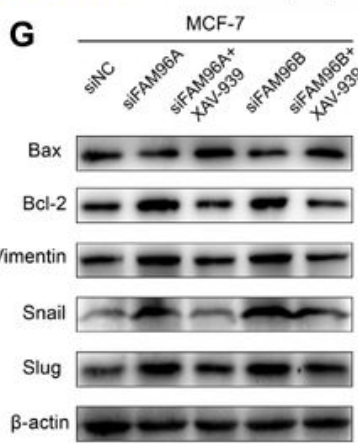

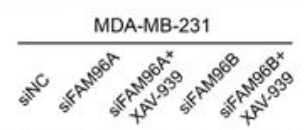

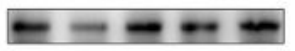

$-0000$

$-0-0$

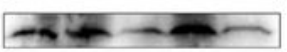

$-\mathrm{e}=\mathrm{er}$

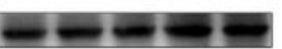

Figure 7 
FAM96A and FAM96B inhibit breast cancer proliferation, invasion and migration through $\mathrm{Wnt} / \beta$-catenin signaling pathway. (A) RT-qPCR analysis of the mRNA levels of Wnt/ $\beta$-catenin signaling pathway related factors including $\beta$-catenin, cyclin D1 and c-Myc in the FAM96A and FAM96B overexpressed and depleted

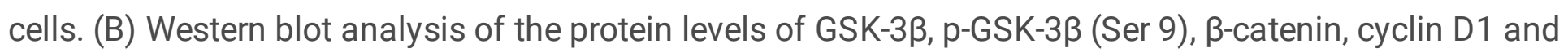
C-Myc in the FAM96A- and FAM96B-overexpressed or -depleted cells. (C) MTT assay for FAM96A- and FAM96B-depleted MCF-7 and MDA-MB-231 cells with or without the specific Wnt/ $\beta$-catenin signaling pathway inhibitor XAV-939 treatment. (D, E) Transwell migration and Matrigel invasion assays for FAM96A and FAM96B-depleted MCF-7 and MDA-MB-231 cells with or without XAV-939 treatment. (F) The

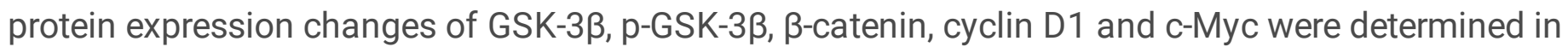
FAM96A and FAM96B-depleted MCF-7 and MDA-MB-231 cells with or without XAV-939 treatment. (G) Cell apoptosis-associated proteins Bax and Bcl-2, as well as EMT-associated marker proteins Vimentin, Snail and Slug were determined by Western blot assay in the absence or presence of XAV-939 treatment. $\beta$ actin was regarded as an internal control. The presented data were the mean \pm SEM of three independent experiments. ${ }^{*} p<0.05,{ }^{*} p<0.01$, and ${ }^{* * *} p<0.001$.

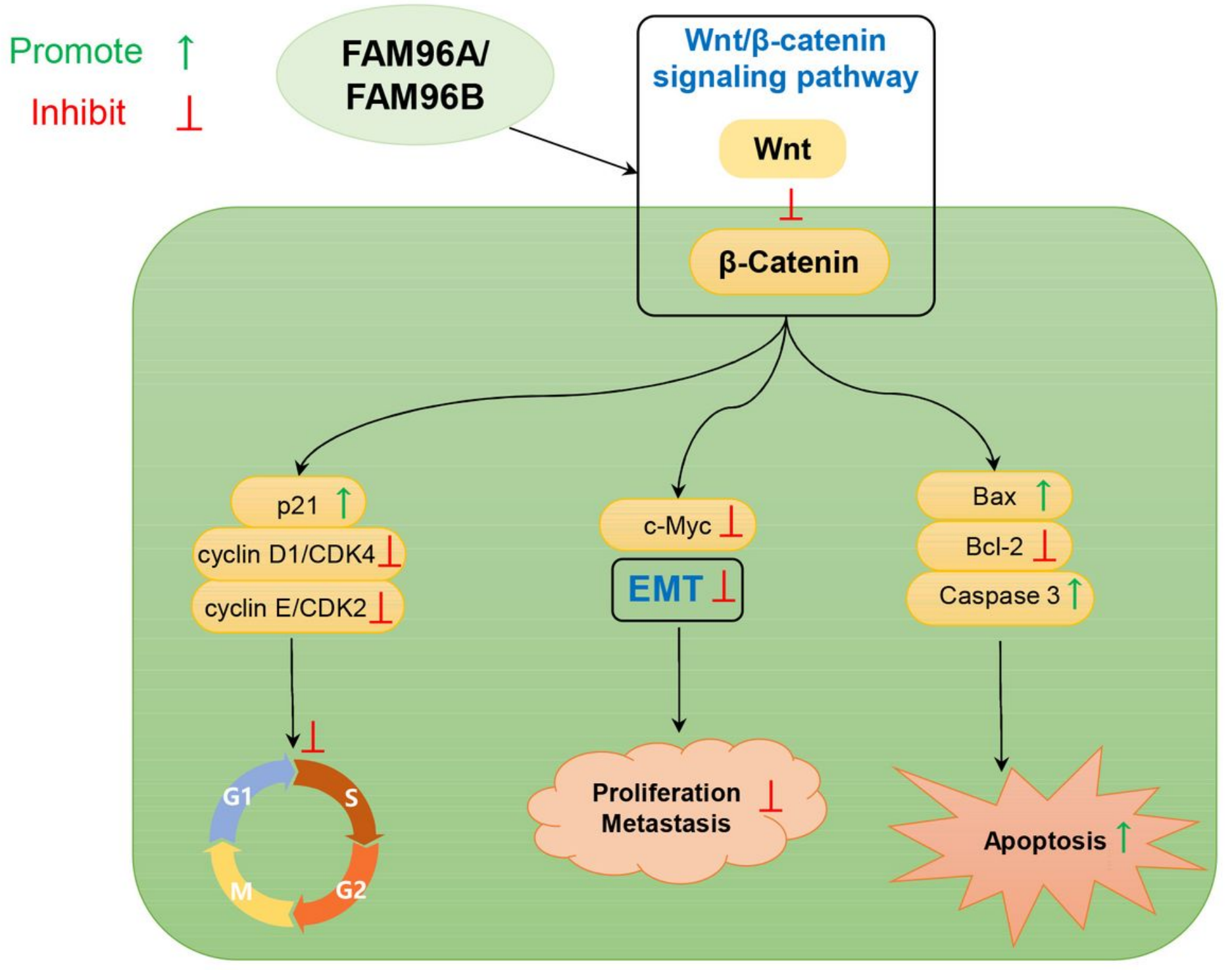




\section{Figure 8}

Schematic diagram depicting the mechanism by which FAM96A and FAM96B inhibit breast cancer cell proliferation, metastasis, block cell cycle and induce apoptosis via the suppression of $\mathrm{Wnt} / \beta$-catenin signaling pathway.

\section{Supplementary Files}

This is a list of supplementary files associated with this preprint. Click to download.

- Supplementarymaterials.docx 